\title{
Approach to equilibrium of a quarkonium in a quark-gluon plasma
}

\author{
Jean-Paul Blaizot $^{1}$ and Miguel Angel Escobedo ${ }^{2}$ \\ ${ }^{1}$ Institut de Physique Théorique, Université Paris Saclay, CEA, CNRS, F-91191 Gif-sur-Yvette, France \\ ${ }^{2}$ Department of Physics, University of Jyväskylä, P.O. Box 35, FI-40014 Jyväskylä, Finland
}

(Received 7 June 2018; published 9 October 2018)

\begin{abstract}
We derive equations of motion for the reduced density matrix of a heavy quarkonium in contact with a quark-gluon plasma in thermal equilibrium. These equations allow in particular a proper treatment of the regime when the temperature of the plasma is comparable to the binding energy of the quarkonium. These equations are used to study how the quarkonium approaches equilibrium with the plasma, and we discuss the corresponding entropy increase, or free energy decrease, depending on the temperature regime. The effect of collisions can be accounted for by the generalization of the imaginary potential introduced in previous studies, and from which collision rates are derived. An important outcome of the present analysis is that this imaginary potential has a sizable dependence on the energy of the relevant transitions.
\end{abstract}

DOI: 10.1103/PhysRevD.98.074007

\section{INTRODUCTION}

There is an ongoing major effort to measure the production of heavy quark bound states in heavy-ion experiments (for a recent review, see Ref. [1]). The goal of such measurements is to obtain information on the medium in which these heavy-quark systems evolve. However, to achieve such a goal, we need to have good control of the dynamics of heavy quarks in a plasma, which is a difficult many-body problem. Different physical effects play a role in modifying the properties of heavy-quark bound states in a quark-gluon plasma, the most prominent ones being the screening of the binding forces and the collisions of the heavy quarks with the plasma constituents. The various models used in phenomenological analyses emphasize one aspect or the other, with of course many refinements in either direction. It is important however that all aspects of the dynamics be treated on the same footing, within a coherent formalism. Only then can we be confident that we understand the processes considered, and eventually extract from the data the properties of the medium in which the bound state evolves.

Important progress in this direction has occurred in the last few years. A major step forward was the recognition that the effect of the collisions could be incorporated in an imaginary potential [2-6], somewhat analogous to the optical potential used in nuclear physics. This imaginary potential can then be calculated, albeit not yet with the

Published by the American Physical Society under the terms of the Creative Commons Attribution 4.0 International license. Further distribution of this work must maintain attribution to the author(s) and the published article's title, journal citation, and DOI. Funded by SCOAP ${ }^{3}$. same degree of accuracy as the real potential that is responsible for binding and is screened in a plasma. Attempts to access it via lattice calculations can be found for instance in Refs. [7,8]. As for the real potential, effective field theories have been used to constrain it in some particular regimes [4-6]. It has also been realized that techniques borrowed from the theory of open quantum systems (see e.g., Ref. [9]) could offer a new perspective on these issues. In particular, the imaginary potential appears naturally in the construction of the operators of the Lindblad equation [10]. The stochastic potential used in some approaches in connection with a Schrödinger equation (see e.g., Refs. [11,12]) is also intimately related to this imaginary potential. As we shall see in this paper, the imaginary potential also directly enters the calculations of the relevant transition rates.

The present paper complements the study presented in Ref. [13]. There, a complete derivation of the equation of motion for the reduced density matrix has been given, under the assumption that the intrinsic dynamics of the heavy quarks is slow compared to that of the plasma. This assumption allowed us to reduce the equations of motion to equations of a Langevin type. This assumption is strictly valid in the regime of high temperature, where the effect of binding forces are small and can be incorporated in the Langevin dynamics. The results that had been obtained along the same lines in the Abelian case in Ref. [14] suggest that it is in this case a reasonable approximation, even when bound states can form. However, this is not so in QCD: when a quarkonium absorbs or emits a gluon its color state changes, from singlet to octet or vice versa. Since the potential between a quark and an antiquark is attractive in the singlet channel, and repulsive in the octet channel, the absorption of a gluon leads to a significant 
change in the effective heavy-quark Hamiltonian. This conflicts with some of the assumptions underlying the derivations presented in Ref. [13], which need therefore to be revisited. More broadly, we need to address more precisely the regime of moderate temperature where the binding energy is of the order of the temperature.

We consider in this paper a simplified setup - a static quark-gluon plasma in thermal equilibrium — and study the time evolution of a single heavy quarkonium in such a medium. The paper contains three main parts. In the next section, we derive equations of motion for the reduced density matrix for a quark-antiquark pair. These equations reproduce in some limit those obtained in Ref. [13], but they lend themselves to more accurate approximations in the regime where the temperature is of the order of the binding energy of the quarkonium. These equations are simplified by integrating out the center-of-mass coordinates, leaving us with equations for the relative motion alone. The second part of the paper, which covers Secs. III and IV, presents a general discussion of how the quarkonium approaches equilibrium with the quark-gluon plasma. We shall see that different treatments can be given depending on whether the temperature is large compared to the binding energy, or comparable to it. This will lead us to consider the variation with time of an (off-equilibrium) entropy and free energy. The third part of the paper, Sec. V, presents some numerical calculations illustrating the main features of the general equations in some simplified situations. Conclusions are summarized at the end.

\section{THE EVOLUTION EQUATION FOR THE DENSITY MATRIX}

We consider a single heavy quark-antiquark pair immersed in a plasma of light quarks and gluons in thermal equilibrium at a temperature $T$ much smaller than the mass $M$ of the heavy quark. The condition $M \gg T$ ensures that we can treat the heavy quark and antiquark as nonrelativistic particles. Also, since the velocity of the heavy particles is small $(\lesssim \sqrt{T / M})$, we neglect their magnetic interactions (among themselves, and with the plasma constituents). ${ }^{1} \mathrm{We}$ assume then that the whole system can be described by the following Hamiltonian:

$$
H=H_{\mathrm{pl}}+H_{Q}+H_{1},
$$

where $H_{\mathrm{pl}}$ is the QCD Hamiltonian governing the dynamics of the plasma while $H_{Q}$ controls the dynamics of the heavy quark-antiquark pair in the absence of the plasma. The Hamiltonian $H_{Q}$ reads

\footnotetext{
${ }^{1}$ This means, in particular, that the processes of gluodissociation are left out of the present discussion. Including those would, however, amount to a straightforward generalization of the present formalism [see e.g., the footnote before Eq. (5.21)].
}

$$
H_{Q}=H_{\mathrm{s}, \mathrm{o}}=-\frac{\Delta_{r}}{M}-\frac{\Delta_{\boldsymbol{R}}}{4 M}+V_{\mathrm{s}, \mathrm{o}}(\boldsymbol{r}),
$$

where $\boldsymbol{r}$ and $\boldsymbol{R}$ denote respectively the relative and the centerof-mass coordinates of the heavy particles. The interaction potential $V_{\mathrm{s}, \mathrm{o}}(\boldsymbol{r})$ is a function of the relative coordinates, and it depends also on the color configuration of the pair. Thus, as indicated in Eq. (2.2), we shall often write $H_{Q}$ as either $H_{\mathrm{s}}$ or $H_{0}$, depending on whether the quark-antiquark pair is in a color singlet $\left(H_{\mathrm{s}}\right)$ or a color octet $\left(H_{\mathrm{o}}\right)$ configuration. In the leading-order nonrelativistic limit, i.e., keeping only the color Coulomb interaction, we have

$$
V_{\mathrm{s}}(\boldsymbol{r})=-\frac{C_{F} \alpha_{s}}{r}, \quad V_{\mathrm{o}}(\boldsymbol{r})=\frac{\alpha_{s}}{2 N_{c} r},
$$

where $C_{F}=\left(N_{c}^{2}-1\right) /\left(2 N_{c}\right)$, where $N_{c}=3$ is the number of colors, and $\alpha_{s}$ is the strong coupling constant, $\alpha_{s}=g^{2} /(4 \pi)$ where $g$ is the gauge coupling.

The last term in Eq. (2.1) is the interaction between the plasma and the heavy quarks. It is of the form ${ }^{2}$

$$
H_{1}=-g \int_{\boldsymbol{x}} a_{0}^{A}(\boldsymbol{x}) n^{A}(\boldsymbol{x}),
$$

where $a_{0}^{A}$ denotes the (color) Coulomb field created by the plasma particles, while $n^{A}$ denotes the color charge density of the heavy particles, where $A$ is a color index. For a quark-antiquark pair, the color charge density is given by

$$
n^{A}(\boldsymbol{x})=\delta(\boldsymbol{x}-\hat{\boldsymbol{r}}) T^{A} \otimes \mathbb{I}-\mathbb{I} \otimes \delta(\boldsymbol{x}-\hat{\boldsymbol{r}}) \tilde{T}^{A},
$$

where $\hat{\boldsymbol{r}}$ denotes the position operator, ${ }^{3}$ and the two components of the tensor product refer respectively to the Hilbert space of the heavy quark (for the first component) and the heavy antiquark (for the second component). In Eq. (2.5), $T^{A}$ is a color matrix in the fundamental representation of SU(3) and describes the coupling between the heavy quark and the gluon. The coupling of the heavy antiquark and the gluon is described by $-\tilde{T}^{A}$, where $\tilde{T}^{A}$ is the transpose of $T^{A}$.

\section{A. The reduced density matrix and its color structure}

Consider now the density matrix $\mathcal{D}$ of the whole system. We assume that initially, at time $t_{0}$, this density matrix factorizes as

$$
\mathcal{D}\left(t_{0}\right)=\mathcal{D}_{Q}\left(t_{0}\right) \otimes \mathcal{D}_{\mathrm{pl}}\left(t_{0}\right)
$$

\footnotetext{
${ }^{2}$ Throughout this paper, we use the shorthand notation $\int_{x} \equiv \int \mathrm{d}^{3} \boldsymbol{x}$ for the spatial integrals, and $\int_{p} \equiv \int \frac{\mathrm{d}^{3} p}{(2 \pi)^{3}}$ for momentum integrals.

${ }^{3}$ We occasionally put a hat on operators whenever confusion may arise from not doing so.
} 
where the plasma density matrix $\mathcal{D}_{\mathrm{pl}}\left(t_{0}\right)$ is an equilibrium density matrix at temperature $T=1 / \beta$ :

$$
\mathcal{D}_{\mathrm{pl}}\left(t_{0}\right)=\frac{\mathrm{e}^{-\beta H}}{Z_{\mathrm{pl}}}, \quad Z_{\mathrm{pl}}=\operatorname{Tre}^{-\beta H_{\mathrm{pl}}} .
$$

The reduced density matrix, $\mathcal{D}_{Q}$, the object that we are mostly concerned with, is defined by taking the trace over the plasma degrees of freedom (d.o.f.)

$$
\mathcal{D}_{Q}(t)=\operatorname{Tr}_{\mathrm{pl}}(\mathcal{D}(t)) .
$$

The state of a heavy quark can be characterized by a position, a color, and a spin. We ignore here the spin d.o.f. Then the reduced density matrix $\mathcal{D}_{Q}$ has matrix elements of the form

$$
\left\langle\boldsymbol{r}_{1} a, \overline{\boldsymbol{r}}_{1} \bar{a}\left|\mathcal{D}_{Q}\right| \boldsymbol{r}_{2} b, \overline{\boldsymbol{r}}_{2} \bar{b}\right\rangle,
$$

where $a, b$ and $\bar{a}, \bar{b}$ are color indices in the fundamental representation and its conjugate, respectively, while $\boldsymbol{r}_{i}$ and $\overline{\boldsymbol{r}}_{i}(i=1,2)$ denote respectively the coordinates of the quark and the antiquark. Factorizing the color structure, one can write $\mathcal{D}_{Q}$ as follows (see Ref. [13] for more details on the color structure of $\mathcal{D}_{Q}$ ):

$$
\begin{aligned}
\mathcal{D}_{Q}(t) & =\left(\frac{\delta_{a \bar{a}} \delta_{b \bar{b}}}{N_{c}} D_{\mathrm{s}}(t)+\frac{T_{a \bar{a}}^{A} T_{\bar{b} b}^{A}}{T_{F}} D_{\mathrm{o}}(t)\right)|a, \bar{a}\rangle\langle b, \bar{b}| \\
& =D_{\mathrm{s}}(t)|\mathrm{s}\rangle\left\langle\mathrm{s}\left|+D_{\mathrm{o}}(t) \sum_{C}\right| \mathrm{o}^{\mathrm{C}}\right\rangle\left\langle\mathrm{o}^{\mathrm{C}}\right|
\end{aligned}
$$

where $D_{\mathrm{s}}$ and $D_{\mathrm{o}}$ are matrices in the two-particle space, with only coordinates as entries, e.g., the matrix elements of $D_{\mathrm{s}}$ are $\left\langle\boldsymbol{r}_{1}, \overline{\boldsymbol{r}}_{1}\left|D_{\mathrm{s}}\right| \boldsymbol{r}_{2}, \overline{\boldsymbol{r}}_{2}\right\rangle$. In the formula above, $T_{F}=1 / 2$ and the color matrices are normalized as $\operatorname{Tr} T^{A} T^{B}=\delta^{A B} / 2$. The relation between the first and second lines of Eq. (2.10) follows from the following formulas:

$$
\langle a \bar{a} \mid \mathrm{s}\rangle=\delta_{a \bar{a}} \frac{1}{\sqrt{N_{c}}}, \quad\left\langle a \bar{a} \mid \mathrm{o}^{\mathrm{C}}\right\rangle=\sqrt{2} T_{a \bar{a}}^{C},
$$

where $|\mathrm{s}\rangle$ and $\left|\mathrm{o}^{C}\right\rangle$ denote respectively color-singlet and -octet (normalized) states, with the index $C$ in $0^{C}$ being a color index that distinguishes the various members of the octet. In the limit where the mass of the heavy quark is infinite, the density matrix is diagonal in coordinate space,

$$
\left\langle\boldsymbol{r}_{1}, \overline{\boldsymbol{r}}_{1}\left|D_{\mathrm{s}}\right| \boldsymbol{r}_{2}, \overline{\boldsymbol{r}}_{2}\right\rangle=\delta\left(\boldsymbol{r}_{1}-\boldsymbol{r}_{2}\right) \delta\left(\overline{\boldsymbol{r}}_{1}-\overline{\boldsymbol{r}}_{2}\right) D_{\mathrm{s}}\left(\boldsymbol{r}_{1}-\overline{\boldsymbol{r}}_{1}\right),
$$

and similarly for $D_{\mathrm{o}}$. In this limit the density matrix depends only on the relative coordinate $\boldsymbol{r}_{1}-\overline{\boldsymbol{r}}_{1}$, which follows from the fact that the plasma in equilibrium is invariant under translations.

\section{B. Approximate evolution equation for the reduced density matrix}

The time evolution of the density matrix of the full system obeys the general equation of motion

$$
i \frac{\mathrm{d} \mathcal{D}}{\mathrm{d} t}=[H, \mathcal{D}] .
$$

In order to treat the interaction between the plasma and the heavy particles by using perturbation theory, we move to the interaction representation with respect to the unperturbed Hamiltonian $H_{0}=H_{Q}+H_{\mathrm{pl}}$ and define

$$
\mathcal{D}(t)=U_{0}\left(t, t_{0}\right) \mathcal{D}^{I}(t) U_{0}^{\dagger}\left(t, t_{0}\right),
$$

where $\mathcal{D}^{I}(t)$ satisfies the equation

$$
\begin{aligned}
\frac{\mathrm{d} \mathcal{D}^{I}}{\mathrm{~d} t} & =-i\left[H_{1}(t), \mathcal{D}^{I}(t)\right], \\
H_{1}(t) & =U_{0}\left(t, t_{0}\right)^{\dagger} H_{1} U_{0}\left(t, t_{0}\right) .
\end{aligned}
$$

We can then rewrite the equation of motion (2.15) as

$$
\frac{\mathrm{d} \mathcal{D}^{I}}{\mathrm{~d} t}=-i\left[H_{1}(t), \mathcal{D}^{I}\left(t_{0}\right)\right]-\int_{t_{0}}^{t} \mathrm{~d} t^{\prime}\left[H_{1}(t),\left[H_{1}\left(t^{\prime}\right), \mathcal{D}^{I}\left(t^{\prime}\right)\right]\right] .
$$

This exact equation is obtained by formally integrating Eq. (2.15) and inserting the obtained solution back into the equation. Perturbation theory at second order in $H_{1}$ is recovered by replacing $\mathcal{D}^{I}\left(t^{\prime}\right)$ by $\mathcal{D}^{I}\left(t_{0}\right)$ in the double commutator.

We can however improve on strict perturbation theory, with the help of two approximations. These are consistent with strict second-order perturbation theory but go beyond, in particular by performing partial resummations (analogous to those in Schwinger-Dyson equations). The first approximation consists in replacing in the double commutator on the right-hand side of Eq. (2.16) the density matrix by the factorized form

$$
\mathcal{D}^{I}(t)=\mathcal{D}_{Q}^{I}(t) \otimes \mathcal{D}_{\mathrm{pl}}^{I}(t) .
$$

This is consistent with second-order perturbation theory since deviation from this form necessarily involves extra powers of $H_{1}$. The factorization allows us to easily perform the average over the plasma d.o.f. We then obtain the following equation for the reduced density matrix of the heavy quarks: 

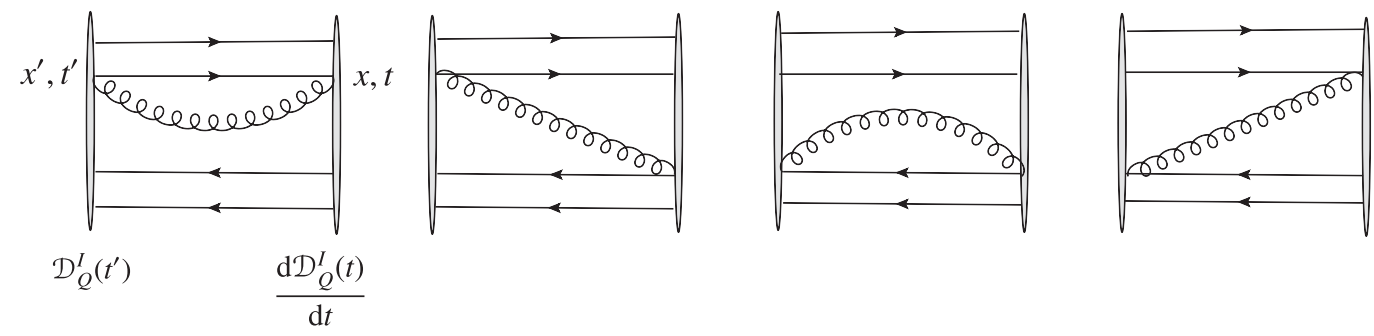

FIG. 1. These four diagrams are in one-to-one correspondence with the four terms in Eq. (2.18). The time flows as in a SchwingerKeldysh contour: forward in the upper part, and backward in the lower part. The two upper lines represent the quark and the antiquark propagating from $t^{\prime}$ to $t$, while the lower lines represent the same particles propagating from $t$ to $t^{\prime}$.

$\frac{\mathrm{d} \mathcal{D}_{Q}^{I}(t)}{\mathrm{d} t}=-g^{2} \int_{t_{0}}^{t} \mathrm{~d} t^{\prime} \int_{\boldsymbol{x} x^{\prime}}\left(\left[n^{A}(t, \boldsymbol{x}), n^{A}\left(t^{\prime}, \boldsymbol{x}^{\prime}\right) \mathcal{D}_{Q}^{I}\left(t^{\prime}\right)\right] \Delta^{>}\left(t-t^{\prime}, \boldsymbol{x}-\boldsymbol{x}^{\prime}\right)+\left[\mathcal{D}_{Q}^{I}\left(t^{\prime}\right) n^{A}\left(t^{\prime}, \boldsymbol{x}^{\prime}\right), n^{A}(t, \boldsymbol{x})\right] \Delta^{<}\left(t-t^{\prime}, \boldsymbol{x}-\boldsymbol{x}^{\prime}\right)\right)$.

We have used the fact that the linear term vanishes in a neutral plasma, and the sum over the color index $A$ is implicit. Finally, we have written the correlator of the $a_{0}$ fields as

$\operatorname{Tr}_{\mathrm{pl}}\left[a_{0}^{A}(t, \mathbf{x}) a_{0}^{B}\left(t^{\prime}, \mathbf{y}\right) \mathcal{D}_{\mathrm{pl}}\right]=\delta^{A B} \Delta^{>}\left(t-t^{\prime}, \mathbf{x}-\mathbf{y}\right)$,

$\operatorname{Tr}_{\mathrm{pl}}\left[a_{0}^{B}\left(t^{\prime}, \mathbf{y}\right) a_{0}^{A}(t, \mathbf{x}) \mathcal{D}_{\mathrm{pl}}\right]=\delta^{A B} \Delta^{<}\left(t-t^{\prime}, \mathbf{x}-\mathbf{y}\right)$.

The equation (2.18) can be given a simple diagrammatic interpretation, illustrated in Fig. 1 (see Ref. [13] for more details). The diagrams involve single gluon exchanges, represented by the correlators (2.19), with the gluon attached at points $\left(t^{\prime}, \boldsymbol{x}^{\prime}\right)$ and $(t, \boldsymbol{x})$. Contributions where the two densities are on the same side of the density matrix in Eq. (2.18), like in $n^{A}(t, \boldsymbol{x}) n^{A}\left(t^{\prime}, \boldsymbol{x}^{\prime}\right) \mathcal{D}_{Q}^{I}\left(t^{\prime}\right)$, are associated to diagrams where the gluon joins lower or upper particle lines among themselves (the first and third diagrams in Fig. 1). Contributions where a density is lying on each side of $\mathcal{D}$, as in $n^{A}(t, \boldsymbol{x}) \mathcal{D}_{Q}^{I}\left(t^{\prime}\right) n^{A}\left(t^{\prime}, \boldsymbol{x}^{\prime}\right)$, are represented by diagrams where the gluon joins upper and lower lines (the second and fourth diagrams in Fig. 1).

The Eq. (2.18) contains a nontrivial memory integral. However a second approximation allows us to obtain a Markovian equation. Indeed, we note that the difference $\mathcal{D}_{Q}^{I}\left(t^{\prime}\right)-\mathcal{D}_{Q}^{I}(t)$ involves powers of the interaction. Thus, at the order at which we are working, we can neglect this difference on the right-hand side of Eq. (2.18), and simply substitute there $\mathcal{D}_{Q}^{I}\left(t^{\prime}\right)$ with $\mathcal{D}_{Q}^{I}(t)$. At this point, the equation still contains a nontrivial time integral, but it is Markovian. Moving back to the Schrödinger picture, one can write this equation as

$$
\begin{aligned}
& \frac{\mathrm{d} \mathcal{D}_{Q}}{\mathrm{~d} t}+i\left[H_{Q}, \mathcal{D}_{Q}(t)\right] \\
& \left.=-g^{2} \int_{x x^{\prime}} \int_{0}^{t-t_{0}} \mathrm{~d} \tau\left[n_{\boldsymbol{x}}^{A}, U_{Q}(\tau) n_{x^{\prime}}^{A} U_{Q}^{\dagger}(\tau) \mathcal{D}_{Q}(t)\right] \Delta^{>}\left(\tau ; \boldsymbol{x}-\boldsymbol{x}^{\prime}\right)\right) \\
& \quad-g^{2} \int_{x x^{\prime}} \int_{0}^{t-t_{0}} \mathrm{~d} \tau\left[\mathcal{D}_{Q}(t) U_{Q}(\tau) n_{x^{\prime}}^{A} U_{Q}^{\dagger}(\tau), n_{x}^{A}\right] \Delta^{<}\left(\tau ; \boldsymbol{x}-\boldsymbol{x}^{\prime}\right),
\end{aligned}
$$

where we have made a change of variable in the time integration, and set $t-t^{\prime}=\tau$. This Markovian equation is the equation that was studied in Ref. [13], and it will prove useful later on. In the rest of this section though, we shall use Eq. (2.18), which has a simpler diagrammatic interpretation. In fact, most of the derivations in this section are blind to this modification of the equation, as will be discussed below. In the Schrödinger picture, Eq. (2.18) reads

$$
\begin{aligned}
\frac{\mathrm{d} \mathcal{D}_{Q}}{\mathrm{~d} t}+i\left[H_{Q}, \mathcal{D}_{Q}(t)\right]= & \left.-g^{2} \int_{x x^{\prime}} \int_{0}^{t-t_{0}} \mathrm{~d} \tau\left[n_{x}^{A}, U_{Q}(\tau) n_{x^{\prime}}^{A} \mathcal{D}_{Q}(t-\tau) U_{Q}^{\dagger}(\tau)\right] \Delta^{>}\left(\tau ; \boldsymbol{x}-\boldsymbol{x}^{\prime}\right)\right) \\
& -g^{2} \int_{x x^{\prime}} \int_{0}^{t-t_{0}} \mathrm{~d} \tau\left[U_{Q}(\tau) \mathcal{D}_{Q}(t-\tau) n_{x^{\prime}}^{A} U_{Q}^{\dagger}(\tau), n_{x}^{A}\right] \Delta^{<}\left(\tau ; \boldsymbol{x}-\boldsymbol{x}^{\prime}\right) .
\end{aligned}
$$

\section{Equation of motion for $D_{\mathrm{s}}$ and $D_{\mathrm{o}}$}

We shall write Eq. (2.21) as follows:

$$
\frac{\mathrm{d}}{\mathrm{d} t} \mathcal{D}_{Q}(t)=-i\left[H, \mathcal{D}_{Q}(t)\right]+\int_{0}^{t-t_{0}} \mathrm{~d} \tau \mathcal{L}(\tau) \mathcal{D}_{Q}(t-\tau),
$$

where $\mathcal{L}$ is to be understood as a linear operator acting on the density matrix. It corresponds typically to one-gluonexchange processes (see Fig. 1), with the gluon being emitted at time $t-\tau$ and absorbed at time $t$. 

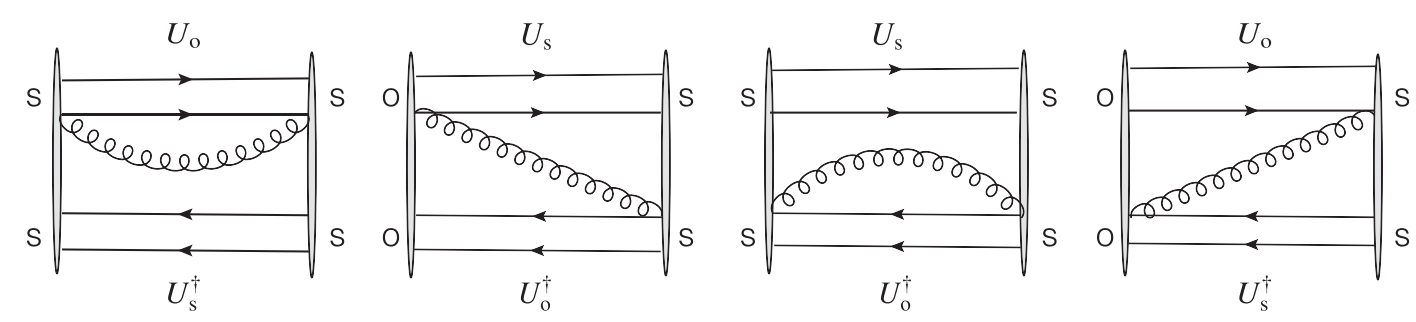

FIG. 2. These four diagrams are in one-to-one correspondence with the four terms in Eqs. (2.28) and (2.29). In these diagrams the evolution operators concern the heavy-quark pair (not the gluon). Each of these diagrams represents four similar diagrams where the gluon is hooked in four possible ways without changing the topological structure [these four contributions are summarized by the propagators $\Delta_{-}^{<}$and $\Delta_{-}^{>}$, cf. Eq. (2.30)]. The first and third diagrams represent $\mathcal{L}^{\text {ss }}$, while the second and fourth represent $\mathcal{L}^{\text {so }}$.

Given the color structure of the density matrix [see Eq. (2.10)], it is convenient to view $\mathcal{L}$ as a matrix in the two-dimensional space spanned by the two components $D_{\mathrm{s}}$ and $D_{\mathrm{o}}$ of the density matrix. Thus, we write

$$
\begin{aligned}
\frac{\mathrm{d} D_{\mathrm{s}}}{\mathrm{d} t}= & -i\left[H_{\mathrm{s}}, D_{\mathrm{s}}\right]+\int_{0}^{t-t_{0}} \mathrm{~d} \tau\left\{\mathcal{L}^{\mathrm{ss}}(\tau) D_{\mathrm{s}}(t-\tau)\right. \\
& \left.+\mathcal{L}^{\mathrm{so}}(\tau) D_{\mathrm{o}}(t-\tau)\right\}, \\
\frac{\mathrm{d} D_{\mathrm{o}}}{\mathrm{d} t}= & -i\left[H_{\mathrm{o}}, D_{\mathrm{o}}\right]+\int_{t_{0}}^{t} d t^{\prime}\left\{\mathcal{L}^{\mathrm{os}}(\tau) D_{\mathrm{s}}(t-\tau)\right. \\
& \left.+\mathcal{L}^{\mathrm{oo}}(\tau) D_{\mathrm{o}}(t-\tau)\right\} .
\end{aligned}
$$

In order to perform the color algebra needed to obtain the explicit expressions of the operators $\mathcal{L}^{i j}$, we note that both the density matrix and the heavy-quark Hamiltonian are diagonal in the singlet-octet basis [a property that we have already used in writing Eq. (2.23)]. Furthermore, we note that the density operator $n^{A}(\boldsymbol{x})$ can connect singlet to octet states, and also various octet states among themselves. Its matrix elements are given by (see e.g., Ref. [13])

$$
\begin{aligned}
\left\langle\mathrm{s}\left|n_{\boldsymbol{x}}^{A}\right| \mathrm{o}^{C}\right\rangle & =\frac{\delta^{A C}}{\sqrt{2 N_{c}}} n(\boldsymbol{x}), \\
\left\langle\mathrm{o}^{D}\left|n_{\boldsymbol{x}}^{A}\right| \mathrm{o}^{C}\right\rangle & =\frac{1}{2} d^{D A C} n(\boldsymbol{x})+\frac{i}{2} f^{D A C} m(\boldsymbol{x}),
\end{aligned}
$$

where

$$
\begin{aligned}
n(\boldsymbol{x}) & \equiv \delta(\boldsymbol{x}-\hat{\boldsymbol{r}}) \otimes \mathbb{I}-\mathbb{I} \otimes \delta(\boldsymbol{x}-\hat{\boldsymbol{r}}), \\
m(\boldsymbol{x}) & \equiv(\boldsymbol{x}-\hat{\boldsymbol{r}}) \otimes \mathbb{I}+\mathbb{I} \otimes \delta(\boldsymbol{x}-\hat{\boldsymbol{r}}) .
\end{aligned}
$$

The calculation then proceeds easily by inserting closure relations into the singlet-octet basis at appropriate places in Eq. (2.21), for instance

$$
\begin{aligned}
\left\langle\mathrm{s}\left|n_{x}^{A} n_{x^{\prime}}^{A}\right| \mathrm{s}\right\rangle= & \sum_{C}\left\langle\mathrm{~s}\left|n_{x}^{A}\right| \mathrm{o}^{C}\right\rangle\left\langle\mathrm{o}^{C}\left|n_{x^{\prime}}^{A}\right| \mathrm{s}\right\rangle, \\
\left\langle\mathrm{o}^{C}\left|n_{x}^{A} n_{x^{\prime}}^{A}\right| \mathrm{o}^{C}\right\rangle= & \left\langle\mathrm{o}^{C}\left|n_{x}^{A}\right| \mathrm{s}\right\rangle\left\langle\mathrm{s}\left|n_{x^{\prime}}^{A}\right| \mathrm{o}^{C}\right\rangle \\
& +\sum_{D}\left\langle\mathrm{o}^{C}\left|n_{x}^{A}\right| \mathrm{o}^{D}\right\rangle\left\langle\mathrm{o}^{D}\left|n_{x^{\prime}}^{A}\right| \mathrm{o} C\right\rangle,
\end{aligned}
$$

and using the following formulas to complete the color algebra:

$$
\begin{aligned}
f^{A B C} f^{A B D}= & N_{c} \delta^{C D}, \quad d^{A B C} d^{A B D}=\frac{N_{c}^{2}-4}{N_{c}} \delta^{C D}, \\
& d^{A B C} \delta^{A B}=0 .
\end{aligned}
$$

It is then straightforward, by taking matrix elements of Eq. (2.21) in singlet or octet states, to obtain the expressions for the operators $\mathcal{L}^{i j}$ in Eq. (2.23). Thus, by taking matrix elements in singlet states, we get (with $t^{\prime} \equiv t-\tau$ )

$$
\begin{aligned}
& \mathcal{L}^{\mathrm{Ss}}(\tau) D_{\mathrm{s}}\left(t^{\prime}\right) \\
&=-g^{2} C_{F} \int_{\mathbf{X}, \mathbf{X}^{\prime}}\left\{\Delta_{-}^{>}\left(X, X^{\prime}\right) \mathcal{P}_{X} U_{\mathrm{o}}(\tau) \mathcal{P}_{X^{\prime}} D_{\mathrm{s}}\left(t^{\prime}\right) U_{\mathrm{s}}^{\dagger}(\tau)\right. \\
&\left.+\Delta_{-}^{\leq}\left(X, X^{\prime}\right) U_{\mathrm{s}}(\tau) D_{\mathrm{s}}\left(t^{\prime}\right) \mathcal{P}_{\boldsymbol{X}^{\prime}} U_{\mathrm{o}}^{\dagger}(\tau) \mathcal{P}_{\boldsymbol{X}}\right\}, \\
& \mathcal{L}^{\mathrm{So}}(\tau) D_{\mathrm{o}}\left(t^{\prime}\right) \\
&=g^{2} C_{F} \int_{\mathbf{X}, \mathbf{X}^{\prime}}\left\{\Delta_{-}^{>}\left(X, X^{\prime}\right) U_{\mathrm{s}}(\tau) \mathcal{P}_{X^{\prime}} D_{\mathrm{o}}\left(t^{\prime}\right) U_{\mathrm{o}}^{\dagger}(\tau) \mathcal{P}_{\boldsymbol{X}}\right. \\
&\left.\quad+\Delta_{-}^{\leq}\left(X, X^{\prime}\right) \mathcal{P}_{\boldsymbol{X}} U_{\mathrm{o}}(\tau) D_{\mathrm{o}}\left(t^{\prime}\right) \mathcal{P}_{\boldsymbol{X}^{\prime}} U_{\mathrm{s}}^{\dagger}(\tau)\right\} .
\end{aligned}
$$

In these equations,

$$
\begin{aligned}
\Delta_{-}^{>}\left(X, X^{\prime}\right)= & \Delta^{>}\left(\tau, \boldsymbol{x}-\boldsymbol{x}^{\prime}\right)+\Delta^{>}\left(\tau, \overline{\boldsymbol{x}}-\overline{\boldsymbol{x}}^{\prime}\right)-\Delta^{>}\left(\tau, \boldsymbol{x}-\overline{\boldsymbol{x}}^{\prime}\right) \\
& -\Delta^{>}\left(\tau, \overline{\boldsymbol{x}}-\boldsymbol{x}^{\prime}\right),
\end{aligned}
$$

and similarly for $\Delta_{-}^{\leq}\left(X, X^{\prime}\right)$. This expression represents the combination of propagators that naturally emerges when one adds the four possible ways to hook the gluon in diagrams with a given topology (i.e., in one of the diagrams of Fig. 2). The minus sign in the last two terms finds its origin in the minus sign present in $n(\boldsymbol{x})$ in Eq. (2.25) and affects the contributions where the gluon couples a quark and an antiquark. Furthermore, we have introduced the notation $X=(t, \boldsymbol{X})$, where $\boldsymbol{X}$ represents the set of coordinates of the quark-antiquark pair, i.e., $\boldsymbol{X}=\{\boldsymbol{x}, \overline{\boldsymbol{x}}\}$, with coordinates without (with) a bar giving the position of the quark (antiquark). The integral $\int_{X}$ in Eq. (2.29) runs over 
these coordinates, i.e., $\int_{\boldsymbol{X}}=\int \mathrm{d}^{3} \boldsymbol{x} \mathrm{d}^{3} \overline{\boldsymbol{x}}$. Finally, $\mathcal{P}_{\boldsymbol{X}}=|\boldsymbol{X}\rangle\langle\boldsymbol{X}|$ is a projector, whose matrix elements between two localized states read

$\left\langle\boldsymbol{r}_{1}, \overline{\boldsymbol{r}}_{1}\left|\mathcal{P}_{\boldsymbol{X}}\right| \boldsymbol{r}_{2}, \overline{\boldsymbol{r}}_{2}\right\rangle=\delta\left(\boldsymbol{r}_{1}-\boldsymbol{r}_{2}\right) \delta\left(\overline{\boldsymbol{r}}_{1}-\overline{\boldsymbol{r}}_{2}\right) \delta\left(\boldsymbol{x}-\boldsymbol{r}_{1}\right) \delta\left(\overline{\boldsymbol{x}}-\overline{\boldsymbol{r}}_{1}\right)$.

The evolution operators now depend on the color state of the propagating quark-antiquark pair. They are $U_{\mathrm{o}}(\tau)=\mathrm{e}^{-i H_{\mathrm{o}} \tau}$ for an octet state and $U_{\mathrm{s}}(\tau)=\mathrm{e}^{-i H_{\mathrm{s}} \tau}$ for a singlet state. The operator $U(\tau)$ propagates the quarkantiquark pair forward in time, i.e., from $t^{\prime}=t-\tau$ to $t$, while its Hermitian conjugate, $U^{\dagger}(\tau)$ propagates the pair backward in time, from $t$ to $t-\tau$. The two operators are therefore attached respectively to the upper and lower pairs of lines in diagrams such as those introduced in Fig. 1. The structure of Eqs. (2.28) and (2.29) may then be understood with the help of the diagrams displayed in Fig. 2.

For the operators $\mathcal{L}^{\mathrm{o} j}$, we get

$$
\mathcal{L}^{\mathrm{os}}(\tau) D_{\mathrm{s}}\left(t^{\prime}\right)=\frac{g^{2}}{2 N_{c}} \int_{\mathbf{X}, \mathbf{X}^{\prime}}\left\{\Delta_{-}^{\geq}\left(X, X^{\prime}\right) U_{\mathrm{o}}(\tau) \mathcal{P}_{\boldsymbol{X}^{\prime}} D_{\mathrm{s}}\left(t^{\prime}\right) U_{\mathrm{s}}^{\dagger}(\tau) \mathcal{P}_{\boldsymbol{X}}+\Delta_{-}^{\leq}\left(X, X^{\prime}\right) \mathcal{P}_{\boldsymbol{X}} U_{\mathrm{s}}(\tau) D_{\mathrm{s}}\left(t^{\prime}\right) \mathcal{P}_{\boldsymbol{X}^{\prime}} U_{\mathrm{o}}^{\dagger}(\tau)\right\}
$$

and, writing $\mathcal{L}^{\mathrm{oo}}=\mathcal{L}_{1}^{\mathrm{oo}}+\mathcal{L}_{2}^{\mathrm{oo}}+\mathcal{L}_{3}^{\mathrm{oo}}$,

$$
\begin{gathered}
\mathcal{L}_{1}^{\mathrm{oo}}(\tau) D_{\mathrm{o}}\left(t^{\prime}\right)=-\frac{g^{2}}{2 N_{c}} \int_{\mathbf{X}, \mathbf{X}^{\prime}}\left\{\Delta_{-}^{>}\left(X, X^{\prime}\right) \mathcal{P}_{X} U_{\mathrm{s}}(\tau) \mathcal{P}_{X^{\prime}} D_{\mathrm{o}}\left(t^{\prime}\right) U_{\mathrm{o}}^{\dagger}(\tau)+\Delta_{-}^{\leq}\left(X, X^{\prime}\right) U_{\mathrm{o}}(\tau) D_{\mathrm{o}}\left(t^{\prime}\right) \mathcal{P}_{X^{\prime}} U_{\mathrm{s}}^{\dagger}(\tau) \mathcal{P}_{\boldsymbol{X}}\right\} \\
\mathcal{L}_{2}^{\mathrm{oo}}(\tau) D_{\mathrm{o}}\left(t^{\prime}\right)=-\frac{g^{2}\left(N_{c}^{2}-4\right)}{4 N_{c}} \int_{\mathbf{X}, \mathbf{X}^{\prime}}\left\{\Delta_{-}^{>}\left(X, X^{\prime}\right)\left[\mathcal{P}_{\boldsymbol{X}}, U_{\mathrm{o}}(\tau) \mathcal{P}_{\boldsymbol{X}^{\prime}} D_{\mathrm{o}}\left(t^{\prime}\right) U_{\mathrm{o}}^{\dagger}(\tau)\right]+\Delta_{-}^{\leq}\left(X, X^{\prime}\right)\left[U_{\mathrm{o}}(\tau) D_{\mathrm{o}}\left(t^{\prime}\right) \mathcal{P}_{\boldsymbol{X}^{\prime}} U_{\mathrm{o}}^{\dagger}(\tau), \mathcal{P}_{\boldsymbol{X}}\right]\right\} \\
\mathcal{L}_{3}^{\mathrm{oo}}(\tau) D_{\mathrm{o}}\left(t^{\prime}\right)=-\frac{g^{2} N_{c}}{4} \int_{\mathbf{X}, \mathbf{X}^{\prime}}\left\{\Delta_{+}^{>}\left(X, X^{\prime}\right)\left[\mathcal{P}_{\boldsymbol{X}}, U_{\mathrm{o}}(\tau) \mathcal{P}_{\boldsymbol{X}^{\prime}} D_{\mathrm{o}}\left(t^{\prime}\right) U_{\mathrm{o}}^{\dagger}(\tau)\right]+\Delta_{+}^{<}\left(X, X^{\prime}\right)\left[U_{\mathrm{o}}(\tau) D_{\mathrm{o}}\left(t^{\prime}\right) \mathcal{P}_{X^{\prime}} U_{\mathrm{o}}^{\dagger}(\tau), \mathcal{P}_{\boldsymbol{X}}\right]\right\}
\end{gathered}
$$

where

$$
\Delta_{+}^{>}\left(X, X^{\prime}\right)=\Delta^{>}\left(\tau, \boldsymbol{x}-\boldsymbol{x}^{\prime}\right)+\Delta^{>}\left(\tau, \overline{\boldsymbol{x}}-\overline{\boldsymbol{x}}^{\prime}\right)+\Delta^{>}\left(\tau, \boldsymbol{x}-\overline{\boldsymbol{x}}^{\prime}\right)+\Delta^{>}\left(\tau, \overline{\boldsymbol{x}}-\boldsymbol{x}^{\prime}\right)
$$

and similarly for $\Delta_{+}^{<}\left(X, X^{\prime}\right)$. Note that there is no minus sign in $\Delta_{+}^{>}\left(X, X^{\prime}\right)$. This is because this contribution arises from products of factors $m(\boldsymbol{x})$ in Eq. (2.25). As for the color factors, their origins can be easily traced back to Eqs. (2.24)-(2.27).

The equations of motion that we have obtained for $D_{\mathrm{s}}$ and $D_{\text {o }}$ are similar to those derived in Ref. [13], to within the small change discussed above [see after Eq. (2.20)], and the fact that in Ref. [13] the Hamiltonian $H_{0}$ used in the interaction representation contains only the heavy quark and antiquark kinetic energy. At that point, in Ref. [13] a further approximation was performed, that consists in expanding the evolution operators at short time, i.e., writing $U(\tau) \simeq 1-i H_{0} \tau$. Here we shall proceed differently in our treatment of the time integrals. The need to go beyond the approximation used in Ref. [13] is motivated in particular by the color-changing transitions that take place in QCD: when a quark-antiquark pair in a singlet state absorbs a gluon, it turns into an octet state. This produces an immediate change in the effective Hamiltonian of the pair, with the force between the quark and the antiquark turning from attractive in the singlet state to repulsive in the octet state. It is then important to keep track of this change of the pair Hamiltonian as the pair propagates during the lifetime of the exchange gluon. This is precisely what the various evolution operators do in the equations written above (see also Figs. 2 and 3), and why we have included the leading-order interaction between the quark and the antiquark in the Hamiltonian $H_{Q}$.

\section{Equations for the relative motion}

At this stage, before doing any further approximation, we shall first simplify the equations that we have obtained by eliminating the center-of-mass coordinates. To that aim, we define a further reduced density matrix by taking a trace over the center-of-mass coordinates. With $\boldsymbol{r}$ and $\overline{\boldsymbol{r}}$ denoting respectively the coordinates of the quark and the antiquark, we call $\boldsymbol{R}=(\boldsymbol{r}+\overline{\boldsymbol{r}}) / 2$ the center-of-mass coordinate and $s=\boldsymbol{r}-\overline{\boldsymbol{r}}$ the relative coordinate. The reduced density matrix is defined from the matrix elements $\left\langle\boldsymbol{r}_{1} \overline{\boldsymbol{r}}_{1}\left|D_{\mathrm{s}, \mathrm{o}}\right| \boldsymbol{r}_{2} \overline{\boldsymbol{r}}_{2}\right\rangle$ which, with a slight abuse of notation, we write also as $\left\langle\boldsymbol{R}_{1}, \boldsymbol{s}_{1}\left|D_{\mathrm{s}, \mathrm{o}}\right| \boldsymbol{R}_{2}, \boldsymbol{s}_{2}\right\rangle$. We call $\tilde{D}_{\mathrm{s}, \mathrm{o}}$ the reduced density matrix obtained after taking the trace over the center-of-mass coordinates. That is, 

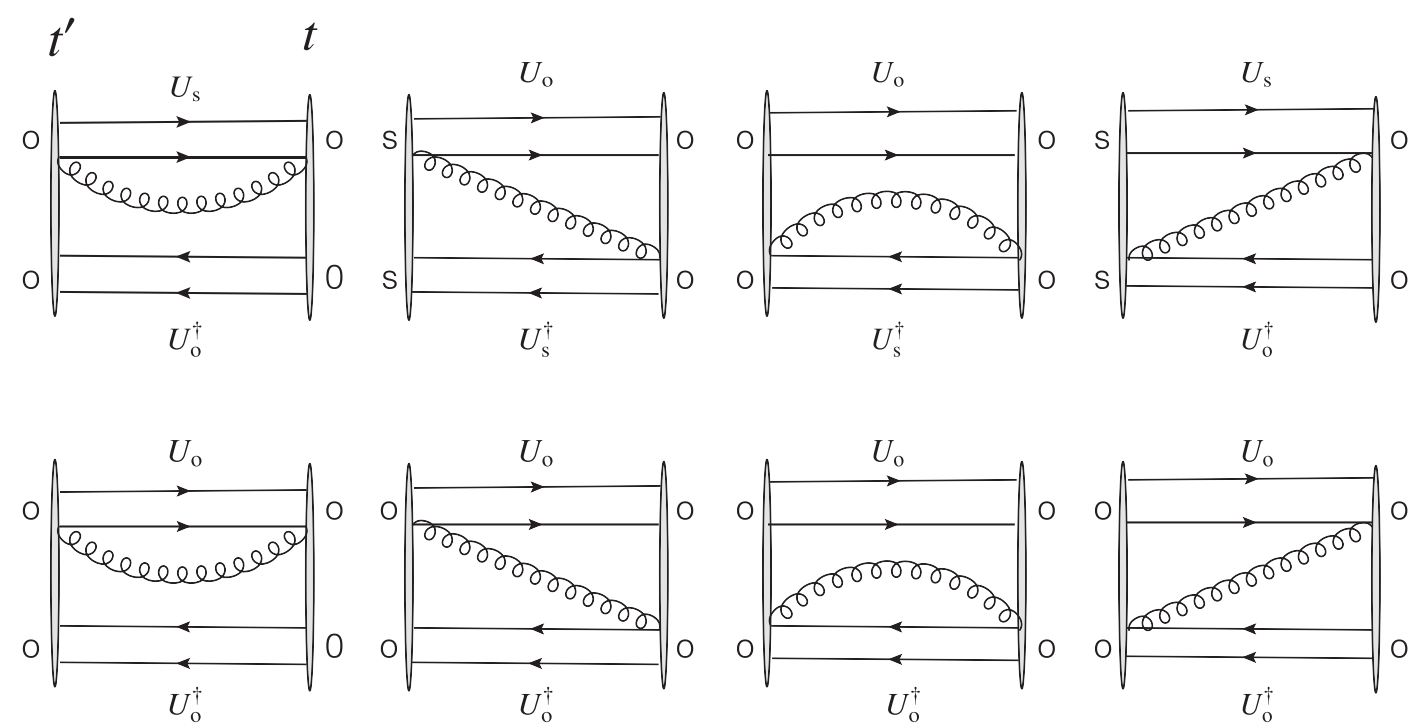

FIG. 3. These four diagrams are in correspondence with the various terms in Eqs. (2.32) and (2.35). For instance the second and fourth diagrams in the first line correspond to $\mathcal{L}^{\text {os }}$, and the first and third correspond to $\mathcal{L}_{1}^{\text {oo }}$, while the diagrams in the second line represent the various contributions to $\mathcal{L}_{2}^{\text {oo }}$ and $\mathcal{L}_{3}^{\text {oo }}$.

$$
\left\langle\boldsymbol{s}_{1}\left|\tilde{D}_{\mathrm{s}, \mathrm{o}}\right| \boldsymbol{s}_{2}\right\rangle \equiv \int_{\boldsymbol{R}}\left\langle\boldsymbol{R}, \boldsymbol{s}_{1}\left|D_{\mathrm{s}, \mathrm{o}}\right| \boldsymbol{R}, \boldsymbol{s}_{2}\right\rangle
$$

The derivation of the equations of motion for the reduced density matrices $D_{\mathrm{s}, \mathrm{o}}$ is presented in Appendix A. It is assumed there that the center-of-mass velocity is small, typically $\lesssim \sqrt{T / M}$. We obtain then the following equations. For the singlet, we get

$$
\mathcal{L}^{\mathrm{ss}}(\tau) \tilde{D}_{\mathrm{s}}\left(t^{\prime}\right)=-g^{2} C_{F}\left\{\Delta^{>}(q) \mathcal{S}_{q \cdot \hat{s}} U_{\mathrm{o}}(\tau) \mathcal{S}_{q \cdot \hat{s}} \tilde{D}_{\mathrm{s}}\left(t^{\prime}\right) U_{\mathrm{s}}(\tau)^{\dagger}+\Delta^{<}(q) U_{\mathrm{s}}(\tau) \tilde{D}_{\mathrm{s}}\left(t^{\prime}\right) \mathcal{S}_{q \cdot \hat{s}} U_{\mathrm{o}}^{\dagger}(\tau) \mathcal{S}_{q \cdot \hat{s}}\right\}
$$

and

$$
\mathcal{L}^{\mathrm{so}}(\tau) \tilde{D}_{\mathrm{o}}(t-\tau)=g^{2} C_{F}\left\{\Delta^{>}(q) U_{\mathrm{s}}(\tau) \mathcal{S}_{q \cdot \hat{s}} \tilde{D}_{\mathrm{o}}\left(t^{\prime}\right) U_{\mathrm{o}}^{\dagger}(\tau) \mathcal{S}_{q \cdot \hat{s}}+\Delta^{<}(q) \mathcal{S}_{q \cdot \hat{\mathrm{s}}} U_{\mathrm{o}}(\tau) \tilde{D}_{\mathrm{o}}\left(t^{\prime}\right) \mathcal{S}_{q \cdot \hat{\mathrm{s}}} U_{\mathrm{s}}^{\dagger}(\tau)\right\}
$$

In these equations (see Appendix A)

$$
\mathcal{S}_{\boldsymbol{q} \cdot \boldsymbol{s}} \equiv 2 \sin (\boldsymbol{q} \cdot \hat{\boldsymbol{s}} / 2)
$$

where $\hat{s}$ is the operator measuring the relative coordinate.

For the octet, we get

$$
\begin{gathered}
\mathcal{L}^{\mathrm{os}}(\tau) \tilde{D}_{\mathrm{s}}\left(t^{\prime}\right)=\frac{g^{2}}{2 N_{c}}\left\{\Delta^{>}(q) U_{\mathrm{o}}(\tau) \mathcal{S}_{q \cdot \hat{s}} \tilde{D}_{\mathrm{s}} U_{\mathrm{s}}^{\dagger}(\tau) \mathcal{S}_{q \cdot \hat{s}}+\Delta^{<}(q) \mathcal{S}_{q \cdot \hat{s}} U_{\mathrm{s}}(\tau) \tilde{D}_{\mathrm{s}}\left(t^{\prime}\right) \mathcal{S}_{q \cdot \hat{s}} U_{\mathrm{o}}^{\dagger}(\tau)\right\}, \\
\mathcal{L}_{1}^{\mathrm{oo}}(\tau) \tilde{D}_{\mathrm{o}}\left(t^{\prime}\right)=-\frac{g^{2}}{2 N_{c}}\left\{\Delta^{>}(q) \mathcal{S}_{q \cdot \hat{s}} U_{\mathrm{s}}(\tau) \mathcal{S}_{q \cdot \hat{s}} \tilde{D}_{\mathrm{o}}\left(t^{\prime}\right) U_{\mathrm{o}}^{\dagger}(\tau)+\Delta^{<}(q) U_{\mathrm{o}}(\tau) \tilde{D}_{\mathrm{o}}\left(t^{\prime}\right) \mathcal{S}_{q \cdot \hat{s}} U_{\mathrm{s}}^{\dagger}(\tau) \mathcal{S}_{q \cdot \hat{s}}\right\}, \\
\mathcal{L}_{2}^{\mathrm{oo}}(\tau) \tilde{D}_{\mathrm{o}}\left(t^{\prime}\right)=-\frac{g^{2}\left(N_{c}^{2}-4\right)}{4 N_{c}}\left\{\Delta^{>}(q)\left[\mathcal{S}_{q \cdot \hat{\mathrm{s}}}, U_{\mathrm{o}}(\tau) \mathcal{S}_{q \cdot \hat{s}} \tilde{D}_{\mathrm{o}}\left(t^{\prime}\right) U_{\mathrm{o}}^{\dagger}(\tau)\right]+\Delta^{<}(q)\left[U_{\mathrm{o}}(\tau) \tilde{D}_{\mathrm{o}}\left(t^{\prime}\right) \mathcal{S}_{q \cdot \hat{s}} U_{\mathrm{o}}^{\dagger}(\tau), \mathcal{S}_{q \cdot \hat{s}}\right]\right\}, \\
\mathcal{L}_{3}^{\mathrm{oo}}(\tau) \tilde{D}_{\mathrm{o}}\left(t^{\prime}\right)=-\frac{g^{2} N_{c}}{4}\left\{\Delta^{>}(q)\left[\mathcal{C}_{q \cdot \hat{s}}, U_{\mathrm{o}}(\tau) \mathcal{C}_{q \cdot \hat{s}} \tilde{D}_{\mathrm{o}}\left(t^{\prime}\right) U_{\mathrm{o}}^{\dagger}(\tau)\right]+\Delta^{<}(q)\left[U_{\mathrm{o}}(\tau) \tilde{D}_{\mathrm{o}}\left(t^{\prime}\right) \mathcal{C}_{q \cdot \hat{s}} U_{\mathrm{o}}^{\dagger}(\tau), \mathcal{C}_{q \cdot \hat{s}}\right]\right\},
\end{gathered}
$$

where we have set $\mathcal{C}_{\boldsymbol{q} \cdot \hat{s}} \equiv 2 \cos (\boldsymbol{q} \cdot \hat{\boldsymbol{s}} / 2)$. 
The equations above give a fairly complete account of the relative motion of a heavy quark-antiquark pair in a static quark-gluon plasma in thermal equilibrium. They are however difficult to solve in full generality. In order to become familiar with their physical content, we consider, in the next two sections the general question of how they describe the approach to thermal equilibrium, in two distinct regimes. The first regime is that of high temperature, controlled by the increase of the entropy. In the second regime, where the temperature is of the order of the binding energy, entropy effects compete with binding forces; there, the relevant quantity to look at is a nonequilibrium free energy which will be seen to decrease monotonously as the equilibrium is approached. We analyze first the Abelian case, and in the following section we consider QCD.

From now on, in order to alleviate the notation, we omit the tilde in $\tilde{D}_{\mathrm{s}, \mathrm{o}}$ since we shall be dealing only with the reduced density matrix of the relative motion.

\section{ENTROPY AND FREE ENERGY IN THE ABELIAN CASE}

A derivation completely analogous to the one done in the previous section gives, for the case of an Abelian plasma,

$$
\frac{\mathrm{d} \mathcal{D}}{\mathrm{d} t}=-i\left[H_{Q}, \mathcal{D}\right]+\int_{0}^{t-t_{0}} \mathrm{~d} \tau \int_{q} e^{-i q_{0} \tau} \mathcal{L}(\tau) \mathcal{D}(t-\tau),
$$

with

$$
\begin{aligned}
\mathcal{L}(\tau) \mathcal{D}(t-\tau)= & -g^{2}\left\{\Delta^{>}(q)\left[\mathcal{S}_{q \cdot \hat{r}}, U(\tau) \mathcal{S}_{q \cdot \hat{r}} \mathcal{D}(t-\tau) U^{\dagger}(\tau)\right]\right. \\
& \left.+\Delta^{<}(q)\left[U(\tau) \mathcal{D}(t-\tau) \mathcal{S}_{q \cdot \hat{r}} U^{\dagger}(\tau), \mathcal{S}_{q \cdot \hat{r}}\right]\right\}
\end{aligned}
$$

This equation is identical to the equation for $D_{\mathrm{s}}$ [Eq. (2.23)] in which we replace $C_{F}$ by unity, set $D_{\mathrm{o}}=D_{\mathrm{s}}=\mathcal{D}$, and use the expressions (2.38) and (2.39). Recall that $\mathcal{D}$ is the reduced density matrix of the quark-antiquark pair, after taking the trace over the center-of-mass coordinates. The operator $\mathcal{S}_{q \cdot \hat{r}}$ is an operator in the space of the relative coordinates [cf. Eq. (2.40)], with $\hat{\boldsymbol{r}}$ denoting the relative coordinate operator. Finally, in $H_{Q}$, the potential is the ordinary Coulomb potential, $V(\boldsymbol{r})=-\alpha / r$ [analogous to $V_{\mathrm{s}}$ in Eq. (2.3) from which it is deduced via the substitutions $C_{F} \rightarrow 1$ and $\alpha_{s} \rightarrow \alpha$, where $\alpha$ is the fine-structure constant].

Our goal now is to perform, approximately, the integration over the time $\tau$, in order to simplify the right-hand side of the equation of motion. The strategies to do so differ, depending on whether we are in the high-temperature limit or not, that is whether binding energy effects play an important role or not.

\section{A. The entropy increase at high temperature}

In this subsection we focus on the high-temperature regime, i.e., the regime where the temperature is much higher than the binding energy. In this regime, one can ignore the binding energy, and the approach to equilibrium is dominated by entropy effects. We shall indeed show that the equations of motion predict a monotonous increase of the entropy. These equations of motion lead naturally to quantum Brownian motion, which was studied more extensively in Ref. [13]. In fact the equation for the reduced density matrix takes the form of a Lindblad equation, where the effects of the collisions are accounted for by an imaginary potential.

The temperature enters the equations through the gluon propagator, and limits the range of the $\tau$ integration to $\tau \lesssim 1 / m_{D}$, with $m_{D} \lesssim T$. ${ }^{4}$ The evolution operators $U(\tau)$ contribute phase factors of the form $\mathrm{e}^{-i \tau H}$, where $H$ is the sum of a kinetic and a potential energy $V$. Collisions change the kinetic energy by an amount $q^{2} / M+P q / M$, where $q \sim m_{D}$ is the typical momentum of a gluon exchanged in a soft collision, and $P \sim \alpha M$ is the typical momentum of the heavy quark or antiquark in its relative motion in a Coulomb bound state. The contribution to the phase factor coming from the change of the kinetic energy is then of order $m_{D} / M \ll 1$ for the first term, and of order $\alpha$ for the second. In either case, these are small contributions that can be safely neglected. As for the binding force acting on the heavy quark, this can be estimated as follows: for a Coulomb bound state, we have parametrically, $\alpha / r \sim \alpha p \sim M \alpha^{2}$. Thus, when $M \alpha^{2} \lesssim m_{D}$, which can be satisfied at sufficiently high temperature, the potential energy $V$, and the binding energy, are small compared to the Debye mass, and one can safely set $U(\tau) \approx 1$ in Eq. (3.2). ${ }^{5}$ Along the same line, we assume that during the time interval between $t-\tau$ and $t$, the density matrix does not vary significantly so that we can replace $\mathcal{D}(t-\tau)$ by $\mathcal{D}(t)$ on the right-hand side of Eq. (3.2) [alternatively, we could use the form (2.20) of the evolution equation for $\mathcal{D}$ ]. With these approximations the evolution equation is greatly simplified, and reads

$$
\begin{aligned}
\frac{\mathrm{d} \mathcal{D}}{\mathrm{d} t}+ & i\left[H_{Q}, \mathcal{D}(t)\right] \\
= & -g^{2} \int_{0}^{t-t_{0}} \mathrm{~d} \tau \int_{q} \mathrm{e}^{-i q_{0} \tau}\left(\Delta^{>}(q)\left[\mathcal{S}_{q \cdot \hat{r}}, \mathcal{S}_{q \cdot \hat{r}} \mathcal{D}(t)\right]\right. \\
& \left.+\Delta^{<}(q)\left[\mathcal{D}(t) \mathcal{S}_{q \cdot \hat{r}}, \mathcal{S}_{q \cdot \hat{r}}\right]\right) .
\end{aligned}
$$

The integrand on the right-hand side can be written as follows:

\footnotetext{
${ }^{4}$ In strict weak coupling, the Debye mass is of order $g T \ll T$. However, it is convenient to relax this condition and allow for values of $m_{D}$ as large as the temperature. In fact, in the numerical calculations presented later, we have typically $m_{D} \simeq 2 T$.

${ }^{5}$ Note that this is a much stronger approximation than that used in Ref. [13] where a linear expansion in $\tau$ was used.
} 


$$
\begin{aligned}
& \frac{1}{2}\left[\mathcal{S}_{q \cdot \hat{r}}^{2}, \mathcal{D}(t)\right]\left(\Delta^{>}(q)-\Delta^{<}(q)\right) \\
& \quad+\left(\frac{1}{2}\left\{\mathcal{S}_{q \cdot \hat{r}}^{2}, \mathcal{D}(t)\right\}-\mathcal{S}_{q \cdot \hat{r}} \mathcal{D}(t) \mathcal{S}_{q \cdot \hat{r}}\right)\left(\Delta^{>}(q)+\Delta^{<}(q)\right)
\end{aligned}
$$

The contributions of the first and the second lines are qualitatively different: as we shall see shortly, the first line yields a correction to the real part of the potential, and corresponds to Hamiltonian evolution, while the second line involves the imaginary part of the potential, and accounts in particular for dissipation. To see that, we perform the integration over $q_{0}$, and then the integration over $\tau$ in Eq. (3.3). In the long-time limit, $t-t_{0} \gg 1 / m_{D}$, we can let the upper limit of the $\tau$ integration go to infinity. The $\tau$ integrations then yield (see Ref. [13])

$$
\begin{gathered}
\int_{0}^{\infty} \mathrm{d} \tau g^{2} \Delta^{>}(\tau ; \boldsymbol{q})=\frac{i}{2} \delta V(\boldsymbol{q})-\frac{1}{2} W(\boldsymbol{q}), \\
\int_{0}^{\infty} \mathrm{d} \tau g^{2} \Delta^{<}(\tau ; \boldsymbol{q})=-\frac{i}{2} \delta V(\boldsymbol{q})-\frac{1}{2} W(\boldsymbol{q}) .
\end{gathered}
$$

The correction $\delta V$ to the real part of the potential provides a contribution that adds up to the Hamiltonian on the left-hand side $\left(H_{Q} \rightarrow H_{Q}^{\prime}\right)$. This reads

$$
\begin{aligned}
-2 i \int_{\boldsymbol{q}} \delta V(\boldsymbol{q})\left[\mathcal{S}_{\boldsymbol{q} \cdot \boldsymbol{r}} \mathcal{S}_{\boldsymbol{q} \cdot \boldsymbol{r}}, \mathcal{D}(t)\right] & =i\left[\int_{\boldsymbol{q}} \delta V(\boldsymbol{q})\left(\mathrm{e}^{i \boldsymbol{q} \cdot \boldsymbol{r}}-1\right), \mathcal{D}\right] \\
& =i[\delta V(\boldsymbol{r})-\delta V(0), \mathcal{D}],
\end{aligned}
$$

where we have used $\delta V(-\boldsymbol{q})=\delta V(\boldsymbol{q})$. This is the screening correction to the real part of the potential. In the hard thermal loop (HTL) approximation, this is given by (see e.g., Ref. [3])

$$
\delta V(r)-\delta V(0)=\frac{\alpha_{s}}{r}\left(\mathrm{e}^{-m_{D} r}-1\right)+\alpha_{s} m_{D},
$$

so that the total potential in $H_{Q}^{\prime}$ is the screened potential $V=\left(\alpha_{s} / r\right) \exp \left(-m_{D} r\right) \quad$ (to within an irrelevant constant term).

The remaining terms can be rearranged as follows:

$$
\begin{aligned}
\frac{\mathrm{d} \mathcal{D}}{\mathrm{d} t} & +i\left[H_{Q}^{\prime}, \mathcal{D}(t)\right] \\
& =-\int_{q} W(\boldsymbol{q})\left(\mathcal{S}_{q \cdot \hat{r}} \mathcal{D}(t) \mathcal{S}_{q \cdot \hat{r}}-\frac{1}{2}\left\{\mathcal{D}(t), \mathcal{S}_{q \cdot \hat{r}}^{2}\right\}\right) .
\end{aligned}
$$

This equation has the structure of a Lindblad equation [15]. It can be written as

$$
\frac{\mathrm{d} \mathcal{D}}{\mathrm{d} t}=-i\left[H_{Q}^{\prime}, \mathcal{D}\right]+\int_{\mathbf{q}}\left(L_{\mathbf{q}} \mathcal{D}(t) L_{\mathbf{q}}^{\dagger}-\frac{1}{2}\left\{\mathcal{D}(t), L_{\mathbf{q}}^{\dagger} L_{\mathbf{q}}\right\}\right),
$$

where the Lindblad operators $L_{\mathbf{q}}$ take the form $\left(g^{2} \Delta^{>}(0, \mathbf{q})=-W(\boldsymbol{q}) \geq 0\right)$

$$
L_{\mathbf{q}}=g \sqrt{\Delta^{>}(0, \mathbf{q})} \mathcal{S}_{\boldsymbol{q} \cdot \boldsymbol{r}}=L_{\mathbf{q}}^{\dagger}
$$

At this point, we could rely on the theorem derived in Ref. [16] for the Lindblad equation, in order to show that the entropy is a monotonically increasing function of time. This theorem requires that $\int \frac{d^{3} q}{(2 \pi)^{3}}\left(L_{\mathbf{q}}^{\dagger} L_{\mathbf{q}}-L_{\mathbf{q}} L_{\mathbf{q}}^{\dagger}\right)=0$, which clearly holds in our case since $L_{\mathbf{q}}^{\dagger}=L_{\mathbf{q}}$. However in order to highlight the difference between the present high-temperature regime, and the low-temperature regime to be discussed in the next subsection, we shall proceed with an explicit and elementary derivation.

From the definition of the von Neumann entropy, ${ }^{6}$

$$
S(t)=-\operatorname{Tr}(\mathcal{D}(t) \log \mathcal{D}(t))
$$

and using the fact that $\operatorname{Tr}(\mathcal{D}(t))$ is independent of time, a property that can be verified explicitly using Eq. (3.10), one easily obtains

$$
\begin{aligned}
\frac{\mathrm{d} S}{\mathrm{~d} t} & =-\operatorname{Tr}\left(\frac{\mathrm{d} \mathcal{D}}{\mathrm{d} t} \log \mathcal{D}\right) \\
& =-\int_{\mathbf{q}} \operatorname{Tr}\left[\left(L_{\mathbf{q}} \mathcal{D}(t) L_{\mathbf{q}}-\frac{1}{2}\left\{\mathcal{D}(t), L_{\mathbf{q}} L_{\mathbf{q}}\right\}\right) \log \mathcal{D}(t)\right] .
\end{aligned}
$$

At this point, it is convenient to use a representation in which $\mathcal{D}(t)$ is diagonal, viz.

$$
\mathcal{D}(t)=\sum_{n} p_{n}(t)|n\rangle\langle n|
$$

with $p_{n}(t) \geq 0$, and the states $n$ are functions of time. We then obtain

\footnotetext{
${ }^{6}$ Since $\mathcal{D}$ is the reduced density matrix, obtained by tracing out the plasma d.o.f., as well as the center-of-mass coordinates, this may also be considered as an entanglement entropy. In this context, it is useful to note that the factorization property (2.17) is used only as an approximation for the right-hand side of the equation of motion (2.16). The resulting equation for the reduced density matrix, Eq. (2.18), keeps track, approximately, of correlations between the heavy quarks and the plasma, and these correlations are responsible for the nontrivial time evolution of the entropy of the heavy-quark system.
} 


$$
\begin{aligned}
\frac{\mathrm{d} S}{\mathrm{~d} t}= & -\int_{\boldsymbol{q}}\left(\sum_{n, m} p_{n}(t) \log p_{m}(t)\left|\left\langle n\left|L_{\mathbf{q}}\right| m\right\rangle\right|^{2}\right. \\
& \left.-\sum_{n} p_{n}(t) \log p_{n}(t)\left\langle n\left|L_{\mathbf{q}} L_{\mathbf{q}}\right| n\right\rangle\right) \\
= & \int_{\boldsymbol{q}} \sum_{n, m}\left|\left\langle n\left|L_{\mathbf{q}}\right| m\right\rangle\right|^{2} p_{n}(t) \log \left(\frac{p_{n}(t)}{p_{m}(t)}\right) \\
= & \frac{1}{2} \int_{\boldsymbol{q}} \sum_{n, m}\left|\left\langle n\left|L_{\mathbf{q}}\right| m\right\rangle\right|^{2}\left[p_{n}(t)-p_{m}(t)\right] \log \left(\frac{p_{n}(t)}{p_{m}(t)}\right) .
\end{aligned}
$$

This expression is manifestly positive, which implies that the entropy (3.12) indeed increases with time. As already emphasized, this proof is less general than the use of the theorem in Ref. [16] (it relies in particular on the property $L_{q}^{\dagger}=L_{q}$ ).

It is interesting to relate the rate of entropy increase to our function $W(\boldsymbol{r})$, or equivalently $\Gamma(\boldsymbol{r})$. A crude estimate can be obtained as follows. First we note that

$$
S(t)=-\sum_{n} p_{n}(t) \log \left(p_{n}(t)\right),
$$

and therefore the combination $\left(p_{n}(t)-p_{m}(t)\right) \log \left(\frac{p_{n}(t)}{p_{m}(t)}\right)$ that appears in Eq. (3.15) is a priori of the same size as $S$. Also, having in mind the approach to equilibrium of a system initially in a bound state, we may write [cf. Eq. (3.11)]

$$
\left\langle n\left|L_{\mathbf{q}}\right| m\right\rangle \approx 2 g \sqrt{\Delta^{>}(0, \mathbf{q})} \sin \left(\frac{\mathbf{q} \mathbf{a}_{0}}{2}\right) A_{n m},
$$

where $\boldsymbol{a}_{0}$ is a vector whose modulus coincides with the Bohr radius of the bound state, and $A_{n m}$ are constants of order unity. We can then estimate the order of magnitude of the change of entropy as follows:

$$
\begin{aligned}
\frac{1 \mathrm{~d} \frac{\mathrm{d}}{S} \mathrm{~d} t}{} & \approx g^{2} \int_{\boldsymbol{q}} \Delta^{>}(0, \mathbf{q})\left(\mathcal{S}_{\boldsymbol{q} \cdot \boldsymbol{a}_{0}}\right)^{2} \\
& =2 \int_{\boldsymbol{q}} W(\boldsymbol{q})\left(\mathrm{e}^{i \boldsymbol{q} \cdot \boldsymbol{a}_{0}}-1\right) \\
& =2 \Gamma\left(a_{0}\right),
\end{aligned}
$$

where we have used $W(\boldsymbol{q})=-g^{2} \Delta^{>}(0, \boldsymbol{q})$, and $\Gamma(\boldsymbol{r})=$ $W(\boldsymbol{r})-W(0)$, where $W(\boldsymbol{r})$ is the imaginary part of the potential. This estimate relates the rate of entropy increase to the imaginary part of the potential, that is to a typical collision rate, at a scale determined by the size of the bound state.

\section{B. Free energy minimization}

Now we look at the regime in which the temperature and the binding energies are of the same order of magnitude. It is no longer legitimate to approximate the evolution operators by unit operators, as we did in the previous subsection.
In this case, the effects of entropy and binding compete. Then, a relevant object to look at is the nonequilibrium generalization of the free energy. To analyze the time dependence of this quantity one is naturally led to expand on a complete set of eigenstates of the Hamiltonian. The equations of motion lead in this case to rate equations, with rates that match those obtained from Fermi's golden rule. One can still express the effect of the collisions, at least partially, through the imaginary part of a potential, provided one takes into account the energy dependence of this potential.

We start again from Eq. (3.1), with the right-hand side written as in Eq. (2.20), that is,

$$
\begin{aligned}
\mathcal{L}(\tau) \mathcal{D}(t-\tau)= & -g^{2}\left\{\Delta^{>}(q)\left[\mathcal{S}_{q \cdot \hat{r}}, U(\tau) \mathcal{S}_{q \cdot \hat{r}} U^{\dagger}(\tau) \mathcal{D}(t)\right]\right. \\
& \left.+\Delta^{<}(q)\left[\mathcal{D}(t) U(\tau) \mathcal{S}_{q \cdot \hat{r}} U^{\dagger}(\tau), \mathcal{S}_{q \cdot \hat{r}}\right]\right\}
\end{aligned}
$$

In order to more easily handle the evolution operators $U(\tau)=\mathrm{e}^{-i H_{Q} \tau}$ and $U^{\dagger}(\tau)$, we introduce at appropriate places projectors on eigenstates of $H_{Q}, \mathcal{P}_{n}=|n\rangle\langle n|$, and assume for simplicity absence of degeneracy. Note that, in contrast to the previous subsection, the states $n$ are now independent of time. We get

$$
\begin{aligned}
\mathcal{L}(\tau) \mathcal{D}(t-\tau) & \\
= & -g^{2} \sum_{n, k}\left\{\Delta^{>}(q) \mathrm{e}^{-i\left(E_{n}-E_{k}\right) \tau}\left[\mathcal{S}_{q \cdot \hat{r}}, \mathcal{P}_{n} \mathcal{S}_{q \cdot \hat{r}} \mathcal{P}_{k} \mathcal{D}(t)\right]\right. \\
& \left.+\Delta^{<}(q) \mathrm{e}^{-i\left(E_{n}-E_{k}\right) \tau}\left[\mathcal{D}(t) \mathcal{P}_{n} \mathcal{S}_{q \cdot \hat{r}} \mathcal{P}_{k}, \mathcal{S}_{q \cdot \hat{r}}\right]\right\}
\end{aligned}
$$

The integration over $\tau$ can then be performed, using

$\int_{0}^{\infty} \mathrm{d} \tau \mathrm{e}^{-i q_{0} \tau} \mathrm{e}^{-i\left(E_{n}-E_{k}\right) \tau} \mathrm{e}^{-\epsilon \tau}=\frac{i}{E_{k}-E_{n}-q_{0}+i \epsilon}$

with $\epsilon \rightarrow 0^{+}$. We get

$$
\begin{aligned}
\frac{\mathrm{d} \mathcal{D}}{d t}+ & i[H, \mathcal{D}] \\
= & -i g^{2} \int_{q} \sum_{k, n}\left(\frac{\Delta^{>}(q)}{E_{k}-E_{n}-q_{0}+i \epsilon}\left[\mathcal{S}_{q \cdot \hat{r}}, \mathcal{P}_{n} \mathcal{S}_{q \cdot \hat{r}} \mathcal{P}_{k} \mathcal{D}(t)\right]\right. \\
& \left.+\frac{\Delta^{<}(q)}{E_{k}-E_{n}-q_{0}+i \epsilon}\left[\mathcal{D}(t) \mathcal{P}_{n} \mathcal{S}_{q \cdot \hat{r}} \mathcal{P}_{k}, \mathcal{S}_{q \cdot \hat{r}}\right]\right)
\end{aligned}
$$

In order to perform the integration over $q_{0}$ we note that

$$
\int_{q_{0}} \frac{\Delta^{>}(q)}{E_{k}-E_{n}-q_{0}+i \epsilon}=-\frac{i}{2} \Delta^{>}\left(E_{k n}, \boldsymbol{q}\right)-\mathrm{P} \int_{q_{0}} \frac{\Delta^{>}\left(q_{0}, \boldsymbol{q}\right)}{q_{0}-E_{k n}},
$$

where we have set $E_{k n} \equiv E_{k}-E_{n}$, and the symbol $\mathrm{P}$ in front of the integral denotes the principal value. Similarly, 


$$
\int_{q_{0}} \frac{\Delta^{<}(q)}{E_{k}-E_{n}-q_{0}+i \epsilon}=\int_{q_{0}} \frac{\Delta^{>}(q)}{E_{k}-E_{n}+q_{0}+i \epsilon}=-\frac{i}{2} \Delta^{>}\left(E_{n k}, \boldsymbol{q}\right)+\mathrm{P} \int_{q_{0}} \frac{\Delta^{>}\left(q_{0}, \boldsymbol{q}\right)}{q_{0}+E_{k n}}
$$

We have used $\Delta^{<}\left(q_{0}, \boldsymbol{q}\right)=\Delta^{>}\left(-q_{0},-\boldsymbol{q}\right)=\Delta^{>}\left(-q_{0}, \boldsymbol{q}\right)$, where the last equality follows from the rotational invariance of the plasma. We can then rewrite Eq. (3.22) as

$$
\begin{aligned}
\frac{\mathrm{d} \mathcal{D}}{d t}+i\left[H_{Q}, \mathcal{D}\right]= & -\frac{g^{2}}{2} \int_{\boldsymbol{q}} \sum_{k, n}\left(\Delta^{>}\left(E_{k n}, \boldsymbol{q}\right)\left[\mathcal{S}_{q \cdot \hat{r}}, \mathcal{P}_{n} \mathcal{S}_{q \cdot \hat{r}} \mathcal{P}_{k} \mathcal{D}(t)\right]+\Delta^{>}\left(E_{n k}, \boldsymbol{q}\right)\left[\mathcal{D}(t) \mathcal{P}_{n} \mathcal{S}_{q \cdot \hat{r}} \mathcal{P}_{k}, \mathcal{S}_{q \cdot \hat{r}}\right]\right) \\
& +i g^{2} \int_{q} \sum_{k, n} \mathrm{P} \int_{q_{0}} \frac{\Delta^{>}\left(q_{0}, \boldsymbol{q}\right)}{q_{0}-E_{k n}}\left[\mathcal{S}_{q \cdot \hat{r}}, \mathcal{P}_{n} \mathcal{S}_{q \cdot \hat{r}} \mathcal{P}_{k} \mathcal{D}(t)\right]-i g^{2} \int_{\boldsymbol{q}} \sum_{k, n} \mathrm{P} \int_{q_{0}} \frac{\Delta^{>}\left(q_{0}, \boldsymbol{q}\right)}{q_{0}+E_{k n}}\left[\mathcal{D}(t) \mathcal{P}_{n} \mathcal{S}_{q \cdot \hat{r}} \mathcal{P}_{k}, \mathcal{S}_{q \cdot \hat{r}}\right] .
\end{aligned}
$$

In the case where the typical transitions involve energy differences that are small compared to the Debye mass, which controls the decay of $\Delta\left(q_{0}\right)$ with $q_{0}$, we can ignore the energies $E_{k n}$, and freely perform the sums over $n$ and $k$, which eliminates the projectors. Using the identities

$$
\mathrm{P} \int_{q_{0}} \frac{g^{2} \Delta^{>}\left(q_{0}, \boldsymbol{q}\right)}{q_{0}}=-\frac{1}{2} \delta V(\boldsymbol{q}), \quad g^{2} \Delta^{>}(0, \boldsymbol{q})=-W(\boldsymbol{q}),
$$

one then easily recovers the result of the previous section, i.e., Eq. (3.9).

We return now to Eq. (3.25). In order to minimize the effects of the principal parts and focus on the dissipative part of the equation, we use the eigenstates of $H_{Q}^{\prime}$ instead of $H_{Q}$, and accordingly subtract the corresponding contribution of $\delta V$ on the right-hand side of the equation. We get

$$
\begin{aligned}
\frac{\mathrm{d} \mathcal{D}}{d t}+i\left[H_{Q}^{\prime}, \mathcal{D}\right]= & -\frac{g^{2}}{2} \int_{\boldsymbol{q}} \sum_{k, n}\left(\Delta^{>}\left(E_{k n}^{\prime}, \boldsymbol{q}\right)\left[\mathcal{S}_{q \cdot \hat{r}}, \mathcal{P}_{n} \mathcal{S}_{q \cdot \hat{r}} \mathcal{P}_{k} \mathcal{D}(t)\right]+\Delta^{>}\left(E_{n k}^{\prime}, \boldsymbol{q}\right)\left[\mathcal{D}(t) \mathcal{P}_{n} \mathcal{S}_{q \cdot \hat{r}} \mathcal{P}_{k}, \mathcal{S}_{q \cdot \hat{r}}\right]\right) \\
& +i g^{2} \int_{q} \sum_{k, n} \mathrm{P} \int_{q_{0}} \Delta^{>}\left(q_{0}, \boldsymbol{q}\right)\left(\frac{1}{q_{0}-E_{k n}^{\prime}}-\frac{1}{q_{0}}\right)\left[\mathcal{S}_{q \cdot \hat{r}}, \mathcal{P}_{n} \mathcal{S}_{q \cdot \hat{r}} \mathcal{P}_{k} \mathcal{D}(t)\right] \\
& -i g^{2} \int_{q} \sum_{k, n} \mathrm{P} \int_{q_{0}} \Delta^{>}\left(q_{0}, \boldsymbol{q}\right)\left(\frac{1}{q_{0}+E_{k n}^{\prime}}-\frac{1}{q_{0}}\right)\left[\mathcal{D}(t) \mathcal{P}_{n} \mathcal{S}_{q \cdot \hat{r}} \mathcal{P}_{k}, \mathcal{S}_{q \cdot \hat{r}}\right],
\end{aligned}
$$

where the energies $E_{n}^{\prime}$ are the eigenvalues of $H_{Q}^{\prime}$. At this point, it is convenient to consider the explicit matrix elements of $\mathcal{D}$ and write the equation in a Liouvillian form

$$
\frac{\mathrm{d} \mathcal{D}_{i j}}{d t}+i E_{i j} \mathcal{D}_{i j}=\mathcal{L}_{i j, k l} \mathcal{D}_{k l}
$$

In order to simplify the writing, we set $\left\langle i\left|\mathcal{S}_{q, \hat{r}}\right| j\right\rangle \rightarrow \mathcal{S}_{i j}$ in the following. We then obtain

$$
\begin{aligned}
\mathcal{L}_{i j, k l}= & -\frac{g^{2}}{2} \int_{\boldsymbol{q}}\left(\mathcal{S}_{\text {in }} \mathcal{S}_{n k} \delta_{j l} \Delta^{>}\left(E_{k n}^{\prime}, \boldsymbol{q}\right)-\mathcal{S}_{i k} \mathcal{S}_{l j} \Delta^{>}\left(E_{k i}^{\prime}, \boldsymbol{q}\right)\right)-\frac{g^{2}}{2} \int_{\boldsymbol{q}}\left(\mathcal{S}_{l n} \mathcal{S}_{n j} \delta_{i k} \Delta^{>}\left(E_{l n}^{\prime}, \boldsymbol{q}\right)-\mathcal{S}_{i k} \mathcal{S}_{l j} \Delta^{>}\left(E_{l j}^{\prime}, \boldsymbol{q}\right)\right) \\
& +i g^{2} \int_{\boldsymbol{q}}\left(\mathcal{S}_{\mathrm{in}} \mathcal{S}_{n k} \delta_{j l} \mathrm{P}^{\prime} \int_{q_{0}} \frac{\Delta^{>}\left(q_{0}, \boldsymbol{q}\right)}{q_{0}-E_{k n}^{\prime}}-\mathcal{S}_{i k} \mathcal{S}_{l j} \mathrm{P}^{\prime} \int_{q_{0}} \frac{\Delta^{>}\left(q_{0}, \boldsymbol{q}\right)}{q_{0}-E_{k i}^{\prime}}\right) \\
& -i g^{2} \int_{\boldsymbol{q}}\left(\mathcal{S}_{l n} \mathcal{S}_{n j} \delta_{i k} \mathrm{P}^{\prime} \int_{q_{0}} \frac{\Delta^{>}\left(q_{0}, \boldsymbol{q}\right)}{q_{0}+E_{n l}^{\prime}}-\mathcal{S}_{i k} \mathcal{S}_{l j} \mathrm{P}^{\prime} \int_{q_{0}} \frac{\Delta^{>}\left(q_{0}, \boldsymbol{q}\right)}{q_{0}+E_{j l}^{\prime}}\right) .
\end{aligned}
$$

In this equation, $\mathrm{P}^{\prime}$ denotes the principal part of the integral from which the contribution $1 / q_{0}$ is subtracted [cf. Eq. (3.27)]. 
Assuming that the Liouvillian on the right-hand side of Eq. (3.28) can be treated as a perturbation, we expect the effect of this perturbation at late time to be dominant when it connects pairs of states with comparable energy differences, that is, when $\left|E_{i j}\right| \simeq\left|E_{k l}\right|$. In particular, one expects the diagonal elements of the density matrix, i.e., the occupation probabilities of the various levels, for which $E_{i j}=0$ to decouple from the nondiagonal ones. ${ }^{7}$ We now restrict ourselves to these diagonal matrix elements, and ignore possible degeneracies. It is easy to verify that the principal values then cancel. We get, for the case $i \neq k$,

$$
\mathcal{L}_{i i, k k}=g^{2}\left|\mathcal{S}_{i k}\right|^{2} \Delta^{>}\left(E_{k i}, \boldsymbol{q}\right) .
$$

This is nothing but the decay rate $\Gamma_{k \rightarrow i}$ calculated according to Fermi's golden rule. That is,

$$
\Gamma_{k \rightarrow i}=g^{2} \int_{\boldsymbol{q}}\left|\left\langle i\left|\mathcal{S}_{q \cdot \hat{r}}\right| k\right\rangle\right|^{2} \Delta^{>}\left(E_{k i}, \boldsymbol{q}\right),
$$

where $\Delta^{>}\left(E_{k i}, \boldsymbol{q}\right)$ plays the role of the density of available states for the transition $k \rightarrow i$. Similarly, for the case $i=k$ we get

$$
\mathcal{L}_{i i, i i}=\sum_{j \neq i} \Gamma_{i \rightarrow j}
$$

At this point we denote by $p_{n}(t)=\langle n|\mathcal{D}(t)| n\rangle$ the probability to find the system in the eigenstate $n$ of $H_{Q}^{\prime}$. The Eq. (3.28) then yields

$$
\frac{\mathrm{d} p_{n}}{\mathrm{~d} t}=\sum_{k}\left(p_{k} \Gamma_{k \rightarrow n}-p_{n} \Gamma_{n \rightarrow k}\right) .
$$

From the property

$$
\Delta^{>}\left(E_{k n}, \mathbf{q}\right)=e^{-\frac{E_{n k}}{T}} \Delta^{>}\left(E_{n k}, \mathbf{q}\right)
$$

and Eq. (3.31), it follows that

$$
\Gamma_{k \rightarrow n}=\mathrm{e}^{-E_{n k} / T} \Gamma_{n \rightarrow k} .
$$

Thus, in equilibrium where $\mathrm{d} p_{n} / \mathrm{d} t=0$, the detailed balance relation $p_{k} \Gamma_{k \rightarrow n}=p_{n} \Gamma_{n \rightarrow k}$ implies

$$
\frac{p_{k}}{p_{n}}=\mathrm{e}^{-\left(E_{k}-E_{n}\right) / T},
$$

that is $p_{n} \propto \mathrm{e}^{-E_{n} / T}$. In other words, the system reaches thermal equilibrium at the temperature of the plasma.

In order to see globally how the equilibrium is achieved, we look at the free energy $F$, defined in terms of the density matrix as in equilibrium, viz.

\footnotetext{
${ }^{7} \mathrm{~A}$ more formal discussion of this issue is presented in Appendix B.
}

$$
F=\operatorname{Tr} H_{Q}^{\prime} \mathcal{D}+T \operatorname{Tr} \mathcal{D} \ln \mathcal{D}=\sum_{n}\left(E_{n}+T \log p_{n}\right) p_{n}
$$

where in the last equality, the states $n$ are the eigenstates of $H_{Q}^{\prime}$. Taking the time derivative of this equation, and using Eqs. (3.33) and (3.34), we get

$$
\begin{aligned}
\frac{\mathrm{d} F}{\mathrm{~d} t} & =-\sum_{n k}\left(E_{n}+T \log p_{n}\right)\left(p_{n}-e^{-\frac{E_{n k}}{T}} p_{k}\right) \Gamma_{n \rightarrow k} \\
& =-\frac{T}{2} \sum_{n k}\left(p_{n}-e^{-\frac{E_{n k}}{T}} p_{k}\right) \log \left(\frac{p_{n}}{p_{k} e^{-\frac{E_{n k}}{T}}}\right) \Gamma_{n \rightarrow k} .
\end{aligned}
$$

Since all the terms in the sum are positive, this equation shows that the free energy is a monotonously decreasing function of time (at least in the large-time limit). An alternative and more formal proof can be obtained by using Lemma 1 of Ref. [17]. Note that if we use this evolution equation to compute the derivatives of $S$ and $E$ separately, one obtains expressions that do not necessarily have a well-defined sign.

We may estimate the rate of change in the free energy, using a similar argument as that used for the entropy. We get

$$
\begin{aligned}
\frac{1}{F} \frac{\mathrm{d} F}{\mathrm{~d} t} & \approx-4 g^{2} \int_{q} \Delta^{>}(\Delta E, \mathbf{q})\left(\sin \left(\frac{\mathbf{q} \mathbf{a}_{0}}{2}\right)\right)^{2} \\
& \approx-2 \Gamma\left(\Delta E, \boldsymbol{a}_{0}\right)
\end{aligned}
$$

where $\Delta E$ is a quantity that represents an average value for the binding energy differences. In the last line $\Gamma\left(\Delta E, a_{0}\right)$ is a damping rate that summarizes the effect of the collisions. This can be viewed as a definition that generalizes that of $\Gamma\left(\boldsymbol{a}_{0}\right)$ to include an energy dependence [i.e., $\Gamma\left(\boldsymbol{a}_{0}\right)=\Gamma\left(\Delta E=0, \boldsymbol{a}_{0}\right)$; see the previous section]. Equation (3.39) is essentially the same as that giving the entropy increase, Eq. (3.18), with $\Delta^{>}(0, \mathbf{q})$ replaced by $\Delta^{>}(\Delta E, \mathbf{q})$. We shall discuss in the last section the size of the correction due to this energy dependence.

\section{ENTROPY AND FREE ENERGY IN A NON-ABELIAN THEORY}

In this section we repeat the analysis of the previous section in the case of QCD. The generalization is straightforward except for obvious complications related to the color algebra, and the existence of several components of the density matrix.

\section{A. Entropy increase}

As in the Abelian case, in the high-temperature limit, the evolution equations for the reduced density matrix are a set of Lindblad equations, in which the effect of collisions is taken into account via an imaginary potential. We shall 
verify that the entropy increases monotonously as the equilibrium is approached.

Following the same reasoning as in Sec. III A we get the following simplified expressions for the various operators $\mathcal{L}$ :

$$
\begin{gathered}
\mathcal{L}^{\mathrm{ss}} D_{\mathrm{s}}=-\frac{C_{F}}{2} \int_{\boldsymbol{q}}\left[L_{\boldsymbol{q}} L_{\boldsymbol{q}}, D_{\mathrm{s}}\right]-\frac{C_{F}}{2} \int_{\boldsymbol{q}}\left\{L_{\boldsymbol{q}} L_{\boldsymbol{q}}, D_{\mathrm{s}}\right\}, \\
\mathcal{L}^{\mathrm{so}} D_{\mathrm{o}}=C_{F} \int_{\boldsymbol{q}} L_{\boldsymbol{q}} D_{\mathrm{o}} L_{\boldsymbol{q}}, \\
\mathcal{L}^{\mathrm{os}} D_{\mathrm{s}}=\frac{1}{2 N_{c}} \int_{\boldsymbol{q}} L_{\boldsymbol{q}} D_{\mathrm{s}} L_{\boldsymbol{q}},
\end{gathered}
$$

and

$$
\begin{gathered}
\mathcal{L}_{1}^{\mathrm{oo}} D_{\mathrm{o}}=-\frac{1}{4 N_{c}} \int_{\boldsymbol{q}}\left[L_{\boldsymbol{q}} L_{\boldsymbol{q}}, D_{\mathrm{o}}\right]-\frac{1}{4 N_{c}} \int_{\boldsymbol{q}}\left\{L_{\boldsymbol{q}} L_{\boldsymbol{q}}, D_{\mathrm{o}}\right\}, \\
\mathcal{L}_{2}^{\mathrm{oo}} D_{\mathrm{o}}=\frac{\left(N_{c}^{2}-4\right)}{4 N_{c}} \int_{\boldsymbol{q}}\left(L_{\boldsymbol{q}} D_{\mathrm{o}} L_{\boldsymbol{q}}-\frac{1}{2}\left\{L_{\boldsymbol{q}} L_{\boldsymbol{q}}, D_{\mathrm{o}}\right\}\right), \\
\mathcal{L}_{3}^{\mathrm{oo}} D_{\mathrm{o}}=\frac{N_{c}}{4} \int_{\boldsymbol{q}}\left(\bar{L}_{\boldsymbol{q}} D_{\mathrm{o}} \bar{L}_{\boldsymbol{q}}-\frac{1}{2}\left\{\bar{L}_{\boldsymbol{q}} \bar{L}_{\boldsymbol{q}}, D_{\mathrm{o}}\right\}\right) .
\end{gathered}
$$

In the previous equations we have used $L_{q}$ as defined in Eq. (3.11) while

$$
\bar{L}_{\boldsymbol{q} \cdot \boldsymbol{r}} \equiv 2 g \sqrt{\Delta^{>}(0, \boldsymbol{q})} \cos (\boldsymbol{q} \cdot \boldsymbol{r} / 2) .
$$

In the QCD case the entropy can be written as

$$
S=-\operatorname{Tr}\left(D_{\mathrm{s}} \log D_{\mathrm{s}}\right)-\left(N_{c}^{2}-1\right) \operatorname{Tr}\left(D_{\mathrm{o}} \log D_{\mathrm{o}}\right),
$$

where the factor $N_{c}^{2}-1$ is due to the normalization chosen in Eq. (2.10). By using the explicit expression of the operators $\mathcal{L}^{i j}$ just given above, we can write the derivative of the entropy as

$$
\begin{aligned}
\frac{\mathrm{d} S}{\mathrm{~d} t}= & -\operatorname{Tr}\left(\left(\mathcal{L}^{\mathrm{ss}} D_{\mathrm{s}}+\mathcal{L}^{\mathrm{so}} D_{\mathrm{o}}\right) \log D_{\mathrm{s}}\right) \\
& -\left(N_{c}^{2}-1\right) \operatorname{Tr}\left(\left(\mathcal{L}^{\mathrm{os}} D_{\mathrm{s}}+\mathcal{L}_{1}^{\mathrm{oo}} D_{\mathrm{o}}\right) \log D_{\mathrm{o}}\right) \\
& -\left(N_{c}^{2}-1\right) \operatorname{Tr}\left(\mathcal{L}_{2}^{\mathrm{oo}} D_{\mathrm{o}} \log D_{\mathrm{o}}\right) \\
& -\left(N_{c}^{2}-1\right) \operatorname{Tr}\left(\mathcal{L}_{3}^{\mathrm{oo}} D_{\mathrm{o}} \log D_{\mathrm{o}}\right) .
\end{aligned}
$$

Using exactly the same reasoning as in Sec. III A we can then show that the second line of Eq. (4.9) is positive, i.e.,

$$
-\left(N_{c}^{2}-1\right) \operatorname{Tr}\left[\left(\mathcal{L}_{2}^{\mathrm{oo}} D_{\mathrm{o}}+\mathcal{L}_{3}^{\mathrm{oo}} D_{\mathrm{o}}\right) \log D_{\mathrm{o}}\right] \geq 0 .
$$

The first line of Eq. (4.9) introduces an additional complication, in particular because it mixes $D_{\mathrm{s}}$ and $D_{\mathrm{o}}$. At this point, we use a (time-dependent) basis in which $D_{\mathrm{s}}$ and $D_{\mathrm{o}}$ are diagonal, that is we set

$$
D_{\mathrm{s}}=\sum_{n} p_{n}^{\mathrm{s}}|\mathrm{s}, n\rangle\left\langle\mathrm{s}, n\left|, \quad D_{\mathrm{o}}=\sum_{m} p_{m}^{\mathrm{o}}\right| \mathrm{o}, m\right\rangle\langle\mathrm{o}, m|,
$$

where, in the last expression, $|\mathrm{o}, m\rangle\langle\mathrm{o}, m|$ actually stands for

$$
|\mathrm{o}, m\rangle\left\langle\mathrm{o}, m\left|=\frac{1}{N_{c}^{2}-1} \sum_{C}\right| \mathrm{o}^{C}, m\right\rangle\left\langle\mathrm{o}^{C}, m\right| .
$$

Then, a simple calculation allows us to write the first line of the right-hand side of Eq. (4.9) as

$$
4 g^{2} C_{F} \sum_{n m}\left(p_{n}^{\mathrm{s}}-p_{m}^{\mathrm{o}}\right) \log \left(\frac{p_{n}^{s}}{p_{m}^{\mathrm{o}}}\right) \int_{q}\left|\left\langle\mathrm{~s}, n\left|L_{\mathbf{q}}\right| \mathrm{o}, m\right\rangle\right|^{2} \geq 0 .
$$

In conclusion, all the terms contributing to the derivative of the entropy are positive. This implies that in the regime where the temperature of the quark-gluon plasma is large in comparison to the typical binding energies, the equations for the reduced density matrix yield a monotonous increase of the entropy as the quarkonium approaches thermal equilibrium. The rate of entropy change can be estimated in the same way as we did for the Abelian case in Sec. III A.

\section{B. Free energy minimization}

We consider now the regime of moderate temperatures, and will proceed to the calculation of the free energy. We shall first write the necessary rate equations describing the evolution of the populations of the various states. Although many of these states belong to a continuum, we write the summations over states as discrete sums, as in Eq. (4.11), instead of integrations, since we focus here on the general structure of the equations. The continuum states will be explicitly dealt with in the examples treated in the next section.

The probabilities $p_{n}^{\mathrm{s}}$ and $p_{m}^{\mathrm{o}}$ fulfill the following evolution equations:

$$
\begin{aligned}
\frac{\mathrm{d} p_{n}^{s}}{\mathrm{~d} t}= & g^{2} C_{F} \sum_{m}\left(p_{m}^{\mathrm{o}}-p_{n}^{\mathrm{s}} e^{-\frac{E_{m}^{o}-E_{n}^{s}}{T}}\right) \\
& \times \int_{\boldsymbol{q}} \Delta^{>}\left(E_{m}^{o}-E_{n}^{s}, \boldsymbol{q}\right)\left|\left\langle\mathrm{s}, n\left|\mathcal{S}_{\boldsymbol{q} \cdot \hat{r}}\right| \mathrm{o}, m\right\rangle\right|^{2},
\end{aligned}
$$

and 


$$
\begin{aligned}
\frac{\mathrm{d} p_{m}^{o}}{\mathrm{~d} t}= & -\frac{g^{2}}{2 N_{c}} \sum_{n}\left(p_{m}^{o}-p_{n}^{s} e^{-\frac{E_{m}^{\mathrm{o}}-E_{n}^{\mathrm{s}}}{T}}\right) \int_{\boldsymbol{q}} \Delta^{>}\left(E_{m}^{\mathrm{o}}-E_{n}^{\mathrm{s}}, \boldsymbol{q}\right)\left|\left\langle\mathrm{s}, n\left|\mathcal{S}_{\boldsymbol{q} \cdot \hat{\boldsymbol{r}}}\right| \mathrm{o}, m\right\rangle\right|^{2} \\
& -\frac{g^{2}\left(N_{c}^{2}-4\right)}{4 N_{c}} \sum_{k}\left(p_{m}^{\mathrm{o}}-p_{k}^{\mathrm{o}} \mathrm{e}^{-\frac{E_{m}^{\mathrm{o}}-E_{k}^{\mathrm{o}}}{T}}\right) \int_{\boldsymbol{q}} \Delta^{>}\left(E_{m}^{\mathrm{o}}-E_{k}^{\mathrm{o}}, \boldsymbol{q}\right)\left|\left\langle\mathrm{o}, m\left|\mathcal{S}_{\boldsymbol{q} \cdot \hat{\boldsymbol{r}}}\right| \mathrm{o}, k\right\rangle\right|^{2} \\
& -\frac{g^{2} N_{c}}{4} \sum_{k}\left(p_{m}^{\mathrm{o}}-p_{k}^{\mathrm{o}} \mathrm{e}^{-\frac{E_{m}^{\mathrm{o}}-E_{k}^{\mathrm{o}}}{T}}\right) \int_{\boldsymbol{q}} \Delta^{>}\left(E_{m}^{\mathrm{o}}-E_{k}^{\mathrm{o}}, \boldsymbol{q}\right)\left|\left\langle\mathrm{o}, m\left|\mathcal{C}_{\boldsymbol{q} \cdot \hat{\boldsymbol{r}}}\right| \mathrm{o}, k\right\rangle\right|^{2} .
\end{aligned}
$$

Note that in order to obtain Eq. (4.14), we had to combine Eqs. (4.1) and (4.2), while the first of Eq. (4.15) involves both Eqs. (4.3) and (4.4). The structure here is very much like what occurs in the entropy calculation, Eq. (4.9).

We may define, in agreement with Fermi's golden rule [see Eq. (3.31)],

$$
\begin{aligned}
& \Gamma_{\mathrm{o}, m \rightarrow \mathrm{s}, n}=\frac{g^{2}}{2 N_{c}} \int_{\boldsymbol{q}} \Delta^{>}\left(E_{m}^{\mathrm{o}}-E_{n}^{\mathrm{s}}, \boldsymbol{q}\right)\left|\left\langle\mathrm{s}, n\left|\mathcal{S}_{\boldsymbol{q} \cdot \hat{r}}\right| \mathrm{o}, m\right\rangle\right|^{2}, \\
& \Gamma_{\mathrm{s}, n \rightarrow \mathrm{o}, m}=g^{2} C_{F} \int_{\boldsymbol{q}} \Delta^{>}\left(E_{n}^{\mathrm{s}}-E_{m}^{\mathrm{o}}, \boldsymbol{q}\right)\left|\left\langle\mathrm{s}, n\left|\mathcal{S}_{\boldsymbol{q} \cdot \hat{\boldsymbol{r}}}\right| \mathrm{o}, m\right\rangle\right|^{2} .
\end{aligned}
$$

The first equation gives the transition rate $\Gamma_{\mathrm{o} \rightarrow \mathrm{s}}$ from one particular member of an octet state to a singlet state [the factor $1 /\left(2 N_{c}\right)$ follows from Eq. (2.24)]. In the second equation, giving $\Gamma_{\mathrm{s} \rightarrow 0}$, all members of the considered octet are summed over (producing a factor $N_{c}^{2}-1$ ). Similarly, we have, for the octet-to-octet transitions

$$
\begin{aligned}
& \Gamma_{\mathrm{o}, m \rightarrow \mathrm{o}, k}^{(2)}=\frac{g^{2}\left(N_{c}^{2}-4\right)}{4 N_{c}} \int_{\boldsymbol{q}} \Delta^{>}\left(E_{m}^{\mathrm{o}}-E_{k}^{\mathrm{o}}, \boldsymbol{q}\right)\left|\left\langle\mathrm{o}, m\left|\mathcal{S}_{\boldsymbol{q} \cdot \hat{\boldsymbol{r}}}\right| \mathrm{o}, k\right\rangle\right|^{2}, \\
& \Gamma_{\mathrm{o}, m \rightarrow \mathrm{o}, k}^{(2)}=\frac{g^{2} N_{c}}{4} \int_{\boldsymbol{q}} \Delta^{>}\left(E_{m}^{\mathrm{o}}-E_{k}^{\mathrm{o}}, \boldsymbol{q}\right)\left|\left\langle\mathrm{o}, m\left|\mathcal{C}_{\boldsymbol{q} \cdot \hat{r}}\right| \mathrm{o}, k\right\rangle\right|^{2} .
\end{aligned}
$$

All these transition rates are those which control the evolution of the populations, according to the equations written above.

With these ingredients we can compute the evolution of the free energy. Expanding on the basis of the eigenstates of $H_{Q}^{\prime}$, and dropping the $/$ on the energies in order to simplify the notation, one gets

$$
\begin{aligned}
F= & \sum_{n}\left(E_{n}^{s} p_{n}^{s}+T p_{n}^{s} \log p_{n}^{s}\right) \\
& +\left(N_{c}^{2}-1\right) \sum_{m}\left(E_{m}^{o} p_{m}^{o}+T p_{m}^{o} \log p_{m}^{o}\right) .
\end{aligned}
$$

Using analogous manipulations as the ones we used in Sec. III B we get

$$
\begin{aligned}
\frac{\mathrm{d} F}{\mathrm{~d} t}= & -T\left(N_{c}^{2}-1\right) \sum_{n m} \log \left(\frac{p_{m}^{o}}{p_{n}^{s} e^{-\frac{E_{m}^{o}-E_{n}^{s}}{T}}}\right) \\
& \times\left(p_{m}^{o}-p_{n}^{s} e^{-\frac{E_{m}^{o}-E_{n}^{s}}{T}}\right) \Gamma_{\mathrm{o}, m \rightarrow \mathrm{s}, n} \\
& -\frac{T}{2} \sum_{m k} \log \left(\frac{p_{m}^{o}}{p_{k}^{o} e^{-\frac{E_{m}^{o}-E_{k}^{o}}{T}}}\right)\left(p_{m}^{o}-p_{k}^{o} e^{-\frac{E_{m}^{o}-E_{k}^{o}}{T}}\right) \Gamma_{\mathrm{o}, m \rightarrow \mathrm{o}, k}^{(2)} \\
& -\frac{T}{2} \sum_{m k} \log \left(\frac{p_{m}^{o}}{p_{k}^{o} e^{-\frac{E_{m}^{o}-E_{k}^{o}}{T}}}\right)\left(p_{m}^{o}-p_{k}^{o} e^{-\frac{E_{m}^{o}-E_{k}^{o}}{T}}\right) \Gamma_{\mathrm{o}, m \rightarrow \mathrm{o}, k}^{(3)} .
\end{aligned}
$$

Again the physical interpretation is straightforward: each of the elementary transitions makes the free energy decrease separately, in a way that is very similar to what happens in the Abelian limit.

\section{SOME ILLUSTRATIVE CALCULATIONS}

In this last section, we present results of some numerical solutions of the equations for the density matrix in simplified situations. We emphasize that our main goal here is to illustrate some of the concepts that we have introduced. Thus, although the numbers are adjusted to bottomonium physics, we make no attempt at a complete phenomenological description. The first example that we treat is that of an infinitely massive quark-antiquark pair. This provides a simple illustration of the role of the energy dependence of the imaginary potential, as well as a quantitative indication of the magnitude of the effect. In principle, such a setting corresponds to that used in lattice QCD calculations, and we briefly compare with relevant lattice results. The next example involves a simplified picture of a bottomonium in a plasma, with a single bound state in the singlet channel, and octet states involving the free quark and antiquark. In this case, rate equations are complemented by a Langevin equation describing the Brownian motion of the heavy quark and antiquark in the plasma.

\section{A. Infinitely massive quark-antiquark pair}

The physics of singlet-to-octet transitions is best analyzed by completely ignoring the motion of the heavy 
particles and focusing on their color d.o.f. alone. This is what we do in this subsection.

We consider an infinitely massive quark-antiquark pair, and assume that it can exist in two color states: a singlet (s) state and a $\left(N_{c}^{2}-1\right)$ degenerate octet (o) state. There are no continuum states, so the problem reduces to that of a twolevel system. The partition function reads

$$
Z=\mathrm{e}^{-\frac{V_{s}}{T}}+\left(N_{c}^{2}-1\right) \mathrm{e}^{-\frac{V_{0}}{T}}
$$

where $V_{\mathrm{s}}(\boldsymbol{r})$ and $V_{\mathrm{o}}(\boldsymbol{r})$ denote the energies of the pair in a singlet and an octet state, respectively. Since the particles do not move, they have no kinetic energy, and the energy of the pair is just the potential energy, which depends on the distance $\boldsymbol{r}$ between the quark and the antiquark. We assume that $V_{\mathrm{s}}<V_{\mathrm{o}}$, and set $\Delta V=V_{\mathrm{o}}-V_{\mathrm{s}}$. The free energy is given by $F=E-T S=-T \ln Z$, where $E$ is the internal energy and $S$ is the entropy. The latter can be deduced from $F$ by using the thermodynamic relation $S=-\partial F / \partial T$. In the low-temperature limit,

$$
F \approx V_{\mathrm{s}}-T\left(N_{c}^{2}-1\right) \mathrm{e}^{-\frac{\Delta V}{T}}
$$

In this regime, the dominant contribution to the free energy is the energy of the ground state $V_{\mathrm{s}}$, the correction from the octet excited states being exponentially small, $\propto T \mathrm{e}^{-\frac{\Delta V}{T}}$. The internal energy and entropy are given by

$$
\begin{aligned}
& E=V_{\mathrm{s}}+\left(N_{c}^{2}-1\right) \Delta V \mathrm{e}^{-\frac{\Delta V}{T}}, \\
& S=\left(N_{c}^{2}-1\right) \mathrm{e}^{-\frac{\Delta V}{T}}\left[1+\frac{\Delta V}{T}\right] .
\end{aligned}
$$

In the opposite limit of high temperature, the free energy is entirely dominated by the entropy. Indeed, a simple calculation yields

$F=-T \ln N_{c}^{2}+\frac{V_{\mathrm{s}}+\left(N_{c}^{2}-1\right) V_{\mathrm{o}}}{N_{c}^{2}}, \quad S=\ln N_{c}^{2}$.

The factor $N_{c}^{2}$ is just the total number of available states: one singlet and $\left(N_{c}^{2}-1\right)$ degenerate octet states. All are present in equilibrium with the same probability. The Boltzmann factors in the partition function can all be approximated by unity, and the internal energy is simply given by

$$
E=\frac{1}{N_{c}^{2}} V_{\mathrm{s}}+\left(1-\frac{1}{N_{c}^{2}}\right) V_{\mathrm{o}}
$$

It is independent of the temperature.

Let $p_{\mathrm{s}}$ and $p_{\mathrm{o}}$ be the probabilities to find the system respectively in the singlet or a given octet state. In the infinite-mass limit, these are simply the diagonal elements of the density matrix [cf. Eq. (2.12)], i.e., $p_{\mathrm{s}}=D_{\mathrm{s}}(\boldsymbol{r})$ and $p_{\mathrm{o}}=D_{\mathrm{o}}(\boldsymbol{r})$. In equilibrium, we have
$p_{\mathrm{s}}=\frac{\mathrm{e}^{-V_{\mathrm{s}} / T}}{Z}, \quad p_{\mathrm{o}}=\frac{\mathrm{e}^{-V_{\mathrm{o}} / T}}{Z}, \quad p_{\mathrm{s}}+\left(N_{c}^{2}-1\right) p_{\mathrm{o}}=1$.

These probabilities depend only on the ratio $\Delta V / T$, which controls the transition between the low- and hightemperature regimes:

$$
\frac{p_{\mathrm{s}}}{p_{\mathrm{o}}}=\mathrm{e}^{\Delta V / T}, \quad p_{\mathrm{s}}=\frac{1}{1+\left(N_{c}^{2}-1\right) \mathrm{e}^{-\Delta V / T}} .
$$

At low temperature, $T \ll \Delta V$, and $p_{\mathrm{s}} \lesssim 1$. At high temperature, $T \gg \Delta V, p_{\mathrm{s}} \simeq p_{\mathrm{o}} \simeq 1 / N_{c}^{2}$. In the numerical calculations, we use

$$
\Delta V(r)=\frac{N_{c} \alpha_{s}(1 / r) e^{-m_{D} r}}{2 r},
$$

where $m_{D}$ is the HTL Debye mass calculated with a running coupling at the scale $2 \pi T$.

We are interested in the dynamics of the approach to the equilibrium state. As we have seen in the previous sections, this is controlled by a rate equation, generically of the form

$$
\frac{\mathrm{d} p_{\mathrm{s}}}{\mathrm{d} t}=\left(N_{c}^{2}-1\right) p_{\mathrm{o}} \Gamma_{\mathrm{o} \rightarrow \mathrm{s}}-p_{\mathrm{s}} \Gamma_{\mathrm{s} \rightarrow \mathrm{o}},
$$

where $\Gamma_{\mathrm{o} \rightarrow \mathrm{s}}$ denotes the transition rate from any one of $N_{c}^{2}-1$ degenerate octet states, and similarly for $\Gamma_{\mathrm{s} \rightarrow \mathrm{o}}$. In a stationary state, the rate equation $\mathrm{d} p_{\mathrm{s}} / \mathrm{d} t=0$ yields the detailed balance condition

$$
\frac{p_{\mathrm{s}}}{p_{\mathrm{o}}}=\frac{\left(N_{c}^{2}-1\right) \Gamma_{\mathrm{o} \rightarrow \mathrm{s}}}{\Gamma_{\mathrm{s} \rightarrow \mathrm{o}}} .
$$

This is to be compared to the result that we expect when the stationary state is the state of thermal equilibrium [cf. Eq. (5.7)]

$$
\frac{p_{\mathrm{s}}(\mathbf{r})}{p_{\mathrm{o}}(\mathbf{r})}=\mathrm{e}^{\frac{\Delta V(\mathbf{r})}{T}}
$$

By comparing the two equations (5.11) and (5.10) one gets the relation

$$
\Gamma_{\mathrm{s} \rightarrow \mathrm{o}} \mathrm{e}^{-V_{\mathrm{s}} / T}=\left(N_{c}^{2}-1\right) \Gamma_{\mathrm{o} \rightarrow \mathrm{s}} \mathrm{e}^{-V_{\mathrm{o}} / T} .
$$

This relation is satisfied by the rates that we have obtained in the previous section. It implies in particular that their energy dependence needs to be taken into account when the temperature is of the same order of magnitude as $\Delta V$; in that case the static imaginary potential is not sufficient to fully account for the effects of collisions. We will return to this issue shortly. 

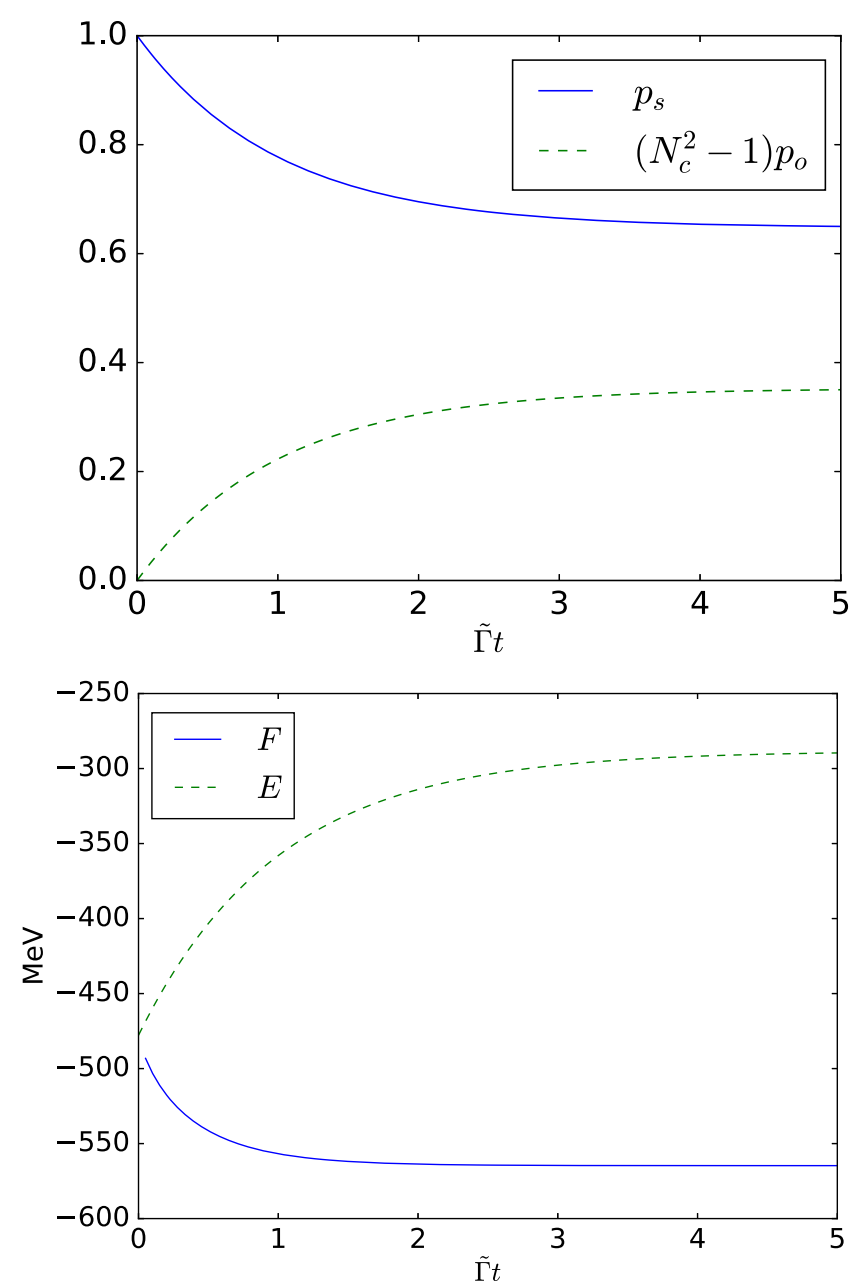

FIG. 4. Top: Time evolution of the probabilities $p_{\mathrm{s}}$ and $p_{\mathrm{o}}$, assuming $p_{\mathrm{s}}(0)=1$ and $r=0.15 \mathrm{fm}$, corresponding approximately to the Bohr radius $a_{0}$ of the singlet bound state. Bottom: Free energy computed in the same conditions. The temperature of the plasma is $T=200 \mathrm{MeV}$.

The evolution equation (5.9) has the following solution, for arbitrary initial conditions:

$$
p_{\mathrm{s}}(t)=p_{\mathrm{s}}^{\mathrm{eq}}\left(1-e^{-\tilde{\Gamma} t}\right)+p_{\mathrm{s}}(0) e^{-\tilde{\Gamma} t}
$$

and

$$
p_{\mathrm{o}}(t)=p_{\mathrm{o}}^{\mathrm{eq}}\left[1+\frac{\mathrm{e}^{\Delta V / T} \mathrm{e}^{-\tilde{\Gamma} t}}{\left(N_{c}^{2}-1\right)}\right]-p_{\mathrm{s}}(0) \frac{\mathrm{e}^{-\tilde{\Gamma} t}}{N_{c}^{2}-1},
$$

where $p_{\mathrm{s}}^{\mathrm{eq}}$ and $p_{\mathrm{o}}^{\mathrm{eq}}$ are the equilibrium values, given in Eq. (5.6), and $\tilde{\Gamma}$ is an effective rate defined as

$$
\tilde{\Gamma}=\Gamma_{\mathrm{s} \rightarrow \mathrm{o}}\left(1+\frac{\mathrm{e}^{\Delta V / T}}{N_{c}^{2}-1}\right)
$$

The solutions $p_{\mathrm{s}}(t)$ and $p_{\mathrm{o}}(t)$ obtained for $p_{\mathrm{s}}(0)=1$ and $r=0.15 \mathrm{fm}$ are plotted in Fig. 4, together with the free energy calculated from Eq. (4.18). For both the survival probability $p_{\mathrm{s}}(t)$ and the free energy, the effective rate $\tilde{\Gamma}(\boldsymbol{r})$ determines the time scale that controls the approach to equilibrium.

In order to quantify the importance of the energy dependence of the rates, we use the explicit results that we have obtained in the previous section. From Eq. (4.14), we get

$$
\frac{d p_{\mathrm{s}}}{d t}=g^{2} C_{F}\left(p_{\mathrm{o}}-e^{-\frac{\Delta V}{T}} p_{\mathrm{s}}\right) \int \frac{d^{3} q}{(2 \pi)^{3}} \Delta^{>}(\Delta V, \mathbf{q})\left(\mathcal{S}_{q \cdot \hat{r}}\right)^{2} .
$$

This equation is identical to Eq. (5.9), now with the following explicit expressions for the rates:

$$
\begin{aligned}
\Gamma_{\mathrm{o} \rightarrow \mathrm{s}} & =\frac{g^{2}}{2 N_{c}} \int_{\boldsymbol{q}} \Delta^{>}(\Delta V, \boldsymbol{q})\left|\mathcal{S}_{\boldsymbol{q} \cdot \hat{r}}\right|^{2}, \\
\Gamma_{\mathrm{s} \rightarrow \mathrm{o}} & =g^{2} C_{F} \int_{\boldsymbol{q}} \Delta^{<}(\Delta V, \boldsymbol{q})\left|\mathcal{S}_{\boldsymbol{q} \cdot \hat{r}}\right|^{2} \\
& =g^{2} C_{F} \mathrm{e}^{-\frac{\Delta V}{T}} \int_{\boldsymbol{q}} \Delta^{>}(\Delta V, \boldsymbol{q})\left|\mathcal{S}_{\boldsymbol{q} \cdot \hat{r}}\right|^{2} .
\end{aligned}
$$

It is easily verified that these rates satisfy Eq. (5.12), as stated above.

In the static limit, and at high temperature, one can express the survival probability of the singlet state in terms of an imaginary potential. We have

$$
\begin{aligned}
\Gamma_{\mathrm{s}}(\boldsymbol{r}) & \equiv C_{F}(W(\boldsymbol{r})-W(0))=C_{F} \int_{q} W(\boldsymbol{q})\left(\mathrm{e}^{i \boldsymbol{q} \cdot \boldsymbol{r}}-1\right) \\
& =\frac{g^{2} C_{F}}{2} \int_{q} \Delta^{<}(0, \mathbf{q})\left(\mathcal{S}_{q \cdot \hat{r}}\right)^{2} \\
& =\frac{1}{2} \Gamma_{\mathrm{s} \rightarrow 0},
\end{aligned}
$$

where we have used $W(\boldsymbol{q})=-g^{2} \Delta^{<}(0, \mathbf{q})$, and in the last line $\Gamma_{\mathrm{s} \rightarrow 0}$ is given by Eq. (5.17) in which one sets $\Delta V=0$. This relation suggests the following definition of a generalized, energy-dependent, "potential" $W(\omega, \boldsymbol{r})$ :

$$
\begin{aligned}
W(\omega, \boldsymbol{r})-W(\omega, 0) & \equiv \frac{g^{2}}{2} \int \frac{d^{3} q}{(2 \pi)^{3}} \Delta^{<}(\omega, \mathbf{q})\left(\mathcal{S}_{q \cdot \hat{r}}\right)^{2} \\
& =\frac{g^{2}}{2} \int \frac{d^{3} q}{(2 \pi)^{3}} N(\omega) \sigma(\omega, \mathbf{q})\left(\mathcal{S}_{q \cdot \hat{r}}\right)^{2} \\
& =g^{2} N(\omega) \int \frac{d^{3} q}{(2 \pi)^{3}} \sigma(\omega, \mathbf{q})\left(1-\mathrm{e}^{i q \cdot \hat{r}}\right)
\end{aligned}
$$

where $\sigma(\omega, \boldsymbol{q})=\Delta^{>}(\omega, \mathbf{q})-\Delta^{<}(\omega, \mathbf{q})$ is the (longitudinal) gluon spectral function, and we have used the relation 
$\Delta^{<}(\omega, \mathbf{q})=\sigma(\omega, \boldsymbol{q}) N(\omega), \quad N(\omega)=\frac{1}{\mathrm{e}^{\omega / T}-1}$.

Note that as $\omega \rightarrow 0, W(\omega, \boldsymbol{r})$ reduces to $W(\boldsymbol{r})$ since, in this limit, $N(\omega) \sim T / \omega$, and $T \sigma(\omega, \boldsymbol{q}) / \omega \rightarrow \Delta^{<}(0, \boldsymbol{q})$.

It is perhaps useful to recall here a few basic properties of the gluon spectral function $\sigma(\omega, \boldsymbol{q})$. To be specific, we shall rely on the HTL approximation, for which an explicit expression is known (see e.g., Ref. [3]). ${ }^{8}$ At fixed momentum, $\sigma(\omega, \boldsymbol{q})$ is an increasing function of the energy (linear at small energy), in the space-like domain $|\omega|<|\boldsymbol{q}|$. For $|\omega|>|\boldsymbol{q}|$ it vanishes, except for an isolated delta-function contribution corresponding to the plasmon excitation at $\omega_{\boldsymbol{q}}$ $\left(\omega_{q}^{2} \simeq \omega_{\mathrm{pl}}^{2}+6 q^{2} / 5\right.$, with $\left.\omega_{\mathrm{pl}}=m_{D} / \sqrt{3}\right)$, which exists only for $|\boldsymbol{q}| \lesssim m_{D}$. The specific contribution of the plasmon to Eq. (5.19) will be ignored in this paper. ${ }^{9}$ For $\omega=0$, we know $\sigma(\omega, \boldsymbol{q})$, and hence $\Delta^{<}(0, \boldsymbol{q})$, analytically. This is

$$
\Delta^{<}(0, \boldsymbol{q})=\frac{\pi m_{D}^{2} T}{|\boldsymbol{q}|\left(\boldsymbol{q}^{2}+m_{D}^{2}\right)^{2}},
$$

so that [2]

$$
\begin{aligned}
\Gamma(\boldsymbol{r})= & W(\boldsymbol{r})-W(0)=\frac{g^{2} T}{2 \pi} \int_{0}^{\infty} \mathrm{d} x \frac{x}{\left(x^{2}+1\right)^{2}} \\
& \times\left[1-\frac{\sin \left(x r m_{D}\right)}{x r m_{D}}\right] .
\end{aligned}
$$

When the energy is nonvanishing, the expression of $\sigma(\omega, \boldsymbol{q})$ is more complicated. It can be obtained from the analytic propagator (see e.g., Ref. [21])

$$
\Delta(\omega, \boldsymbol{q})=-\frac{1}{\boldsymbol{q}^{2}+\Pi_{L}(\omega, \boldsymbol{q})}+\frac{1}{\boldsymbol{q}^{2}}=\int_{q_{0}} \frac{\sigma(\omega, \boldsymbol{q})}{q_{0}-\omega}
$$

where $\Pi_{L}$ is the longitudinal self-energy

$$
\Pi_{L}(\omega, \boldsymbol{q})=m_{D}^{2}\left(1-\frac{\omega}{2 q} \ln \left(\frac{\omega+q}{\omega-q}\right)\right) .
$$

The imaginary part of $\Pi_{L}$ (obtained by setting $\omega \rightarrow \omega+i \eta$, with $\omega$ real) determines the continuum part of the spectral function at small energy. It is given by

\footnotetext{
${ }^{8}$ Note that all the numerical calculations presented in this paper use this approximation. Note also that we shall be using this approximation beyond its strict regime of validity, which requires $\omega, q \ll T$.

${ }^{9}$ It should of course be included in a more quantitative study. It represents a process analogous to that of gluon dissociation involving the transverse modes of the gluon [18-20]. The collective plasmon exists only at small momentum $q \lesssim m_{D}$, and its contribution to $W(\omega, \boldsymbol{r})-W(\omega, 0)$ is expected to be modest in the region of interest. Taking it into account would not alter the main conclusions of this section.
}
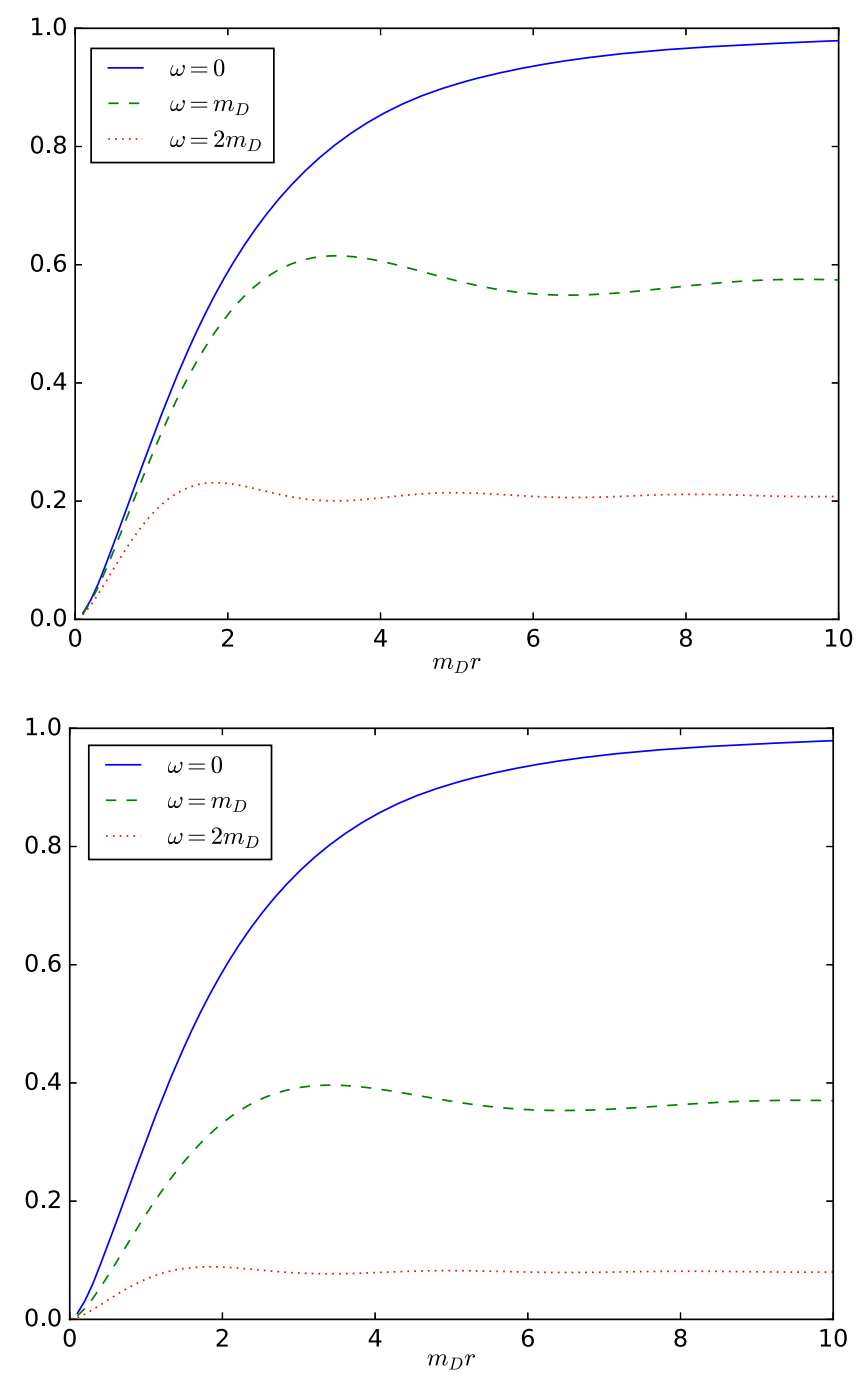

FIG. 5. The function $\left(4 \pi / T g^{2}\right)(W(\omega, r)-W(\omega, 0))$ as a function of $r m_{D}$ for two different temperatures. Top: $T=5 m_{D}$. Bottom: $T=m_{D}$.

$$
\operatorname{Im}_{L}(\omega, \boldsymbol{q})=\frac{\pi m_{D}^{2} \omega}{2 q} \theta(q-|\omega|) .
$$

More generally, we have

$\sigma(\omega, \boldsymbol{q})=\frac{2 \operatorname{Im}_{L}(\omega, \boldsymbol{q})}{\left(\boldsymbol{q}^{2}+\operatorname{Re} \Pi_{L}(\omega, \boldsymbol{q})\right)^{2}+\left(\operatorname{Im} \Pi_{L}(\omega, \boldsymbol{q})\right)^{2}}$.

Note that the temperature enters the spectral function only through the Debye mass $m_{D}$, and we can set $\sigma(\omega, \boldsymbol{q})=m_{D}^{-2} \bar{\sigma}\left(\omega / m_{D}, \boldsymbol{q} / m_{D}\right)$, where $\bar{\sigma}\left(\omega / m_{D}, \boldsymbol{q} / m_{D}\right)$ is a dimensionless function. For later convenience we define the dimensionless quantities $\bar{q}=\frac{q}{m_{D}}$ and $\bar{w}=\frac{w}{m_{D}}$. On the other hand, the statistical factor that multiplies $\sigma(\omega, \boldsymbol{q})$ in Eq. (5.20) depends only on $T$.

Knowing the spectral function, one can then determine the energy-dependent potential (5.19). The results of this calculation are displayed in Fig. 5 for two values of the 
temperature: $T=m_{D}$ and $T=5 m_{D}$. One sees there that the dominant effect of the energy dependence is a sizable reduction of the imaginary potential, a reduction which gets amplified as the temperature, when it is of the order of the energy, decreases. This reduction arises from the fact that as the energy $\omega$ of the transitions increases the phase space of the space-like gluons that induce such transitions decreases. The density of such gluons in momentum space is essentially the quantity $\Delta^{<}(\omega, \boldsymbol{q})$ and its decrease with increasing $\omega$, for a given $q$, results from the combination of two effects: the statistical factor suppresses transitions with $\omega>T$, and the spectral density vanishes when $\omega>q$.

To proceed further, it is convenient to rewrite Eq. (5.19) in terms of dimensionless variables, as follows:

$$
\frac{W(\omega, \boldsymbol{r})-W(\omega, 0)}{g^{2} T}=h\left(\frac{\omega}{T}\right) \frac{1}{\bar{\omega}} \int \frac{d^{3} \bar{q}}{(2 \pi)^{3}} \bar{\sigma}(\bar{\omega}, \overline{\mathbf{q}})\left(1-\mathrm{e}^{\mathrm{i} \overline{\boldsymbol{q}} \cdot \overline{\boldsymbol{r}}}\right),
$$

where

$$
h(x) \equiv \frac{x}{e^{x}-1} .
$$

The curves in Fig. 5 are obtained after integration over $q$, which affects the dependence on $r$ of $W(\omega, r)$ at fixed $\omega$. In particular, at large values of $r m_{D}$, the last term in the integral (5.27) yields a vanishing contribution, which is the origin of the flat behavior observed in Fig. 5. Another factor determines the $r$ dependence of the rates: the energy $\omega$ is to be set equal to $\Delta V(r)$ [see Eq. (5.17)]. It turns out that, after this substitution, the dominant $\boldsymbol{r}$ dependence, in the relevant range, is captured by the function $h$ in Eq. (5.27), that is

$$
\begin{aligned}
W(\Delta V, \boldsymbol{r})-W(\Delta V, 0) & \simeq h\left(\frac{\Delta V}{T}\right) g(\omega=0, \mathbf{r}) \\
& \simeq h\left(\frac{\Delta V}{T}\right) \Gamma_{\mathrm{s}}(\boldsymbol{r}),
\end{aligned}
$$

where, after reinstating the appropriate color factor $C_{F}$, we have set

$$
g(\omega, \mathbf{r}) \equiv \frac{g^{2} C_{F} T}{2 \omega} \int_{\boldsymbol{q}} \sigma(\omega, \boldsymbol{q})\left(\mathcal{S}_{\boldsymbol{q} \cdot \hat{r}}\right)^{2}
$$

and $\Gamma_{\mathrm{s}}(r)$ is given explicitly in Eq. (5.18).

The suppression factor $h\left(\frac{\Delta V(r)}{T}\right)$ is plotted as a function of $r$ for different temperatures in Fig. 6. Note that $h(x) \rightarrow 1$ as $x \rightarrow 0$, while, $h(x) \sim \mathrm{e}^{-x}$ as $x \rightarrow \infty$. Thus, the strong suppression at small $r$ originates from the fact that $\Delta V(r) \rightarrow \infty$ as $r \rightarrow 0$, that is, $h(r) \sim \exp \{-\Delta V(r) / T\}$. This overwhelms the suppression already present in

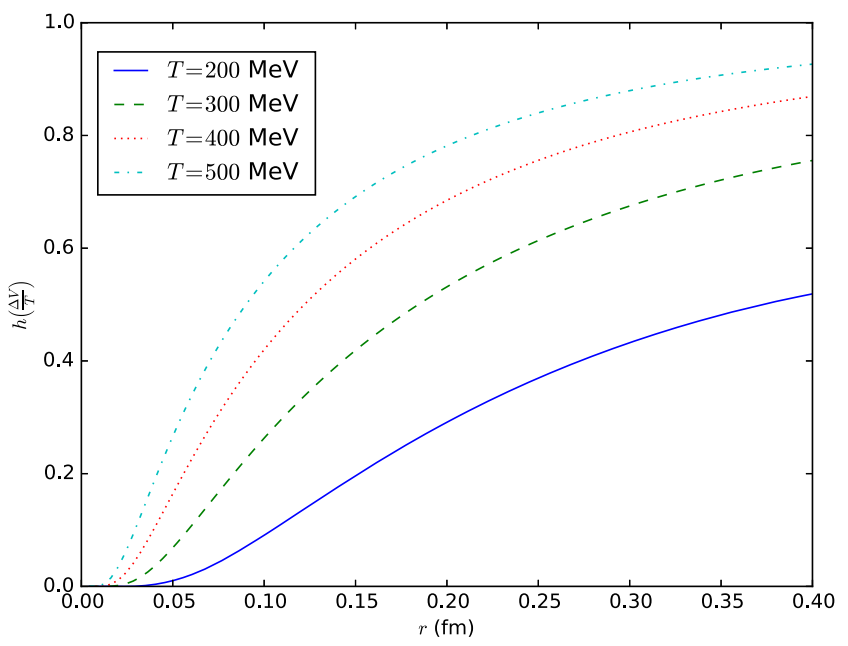

FIG. 6. The function $h\left(\frac{\Delta V}{T}\right)$ vs $r$ for different temperatures.

$\Gamma_{\mathrm{S}}(r) \sim r^{2} \ln (1 / r)$, reflecting color transparency, i.e., the suppression of interactions when the size of the color dipole made by the quark and the antiquark in a color singlet vanishes. At large $r, \Delta V(r) \rightarrow 0$, and $h(r) \sim$ $1-\Delta V(r) /(2 T)$.

The setup discussed in this subsection is very close to that used in lattice QCD calculations of the potential, or free energy, of a heavy quark-antiquark pair. In particular, we may attempt a comparison with the recent results of Ref. [8]. Since the potential calculated there is reconstructed from the spectral function, it should contain, in principle, the energy dependence that we have been discussing. A comparison with the lattice results shows that, as is the case with the imaginary potential that we calculate, the small $r$ dependence is clearly different from the behavior $\left(\sim-r^{2} \log (r)\right)$ expected in the absence of energy dependence: there is a strong suppression at

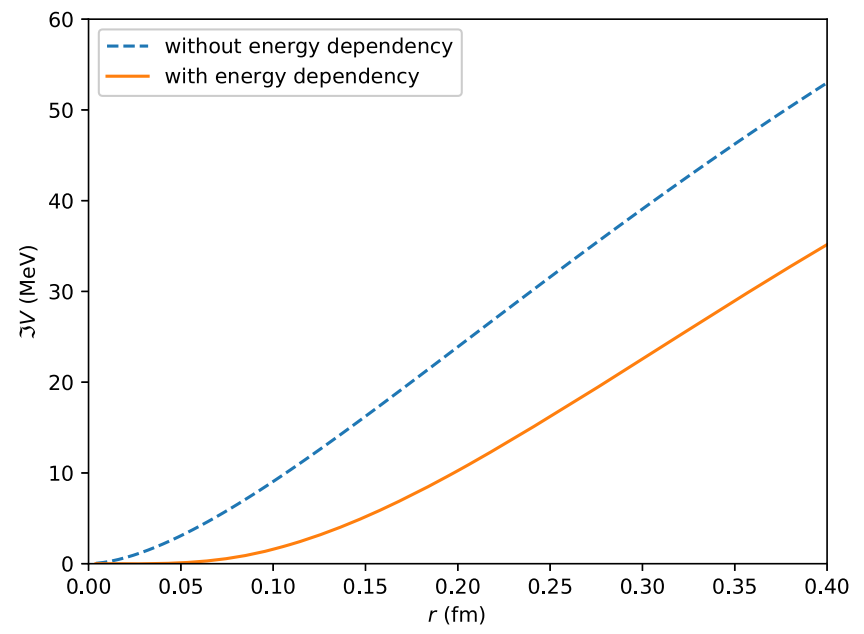

FIG. 7. Comparison of the imaginary potential $(W(r)-W(0))$ with and without energy dependence, at $T=250 \mathrm{MeV}$. 
small distances which continues up to larger radii as the temperature decreases. Unfortunately, a more quantitative comparison between our computation and the lattice simulations is difficult, since the Coulomb approximation that we use is not accurate at large distance, and an additional effect due to the string tension cannot be excluded, as discussed in Ref. [8].

Finally, we return to the estimates of the rate of entropy or free energy variations for which expressions were derived in Sec. III. The explicit expression of $\Gamma(\boldsymbol{r})$ in the HTL approximation is given in Eq. (5.22). Using this approximation we estimate that at $T=250 \mathrm{MeV}$, and using $a_{0}=\frac{1}{1200} \mathrm{MeV}^{-1}$ for the Bohr radius, the time scale that characterizes the changes in the entropy is around $12 \mathrm{fm} / \mathrm{c}$. This is of the order of the typical total lifetime of the fireball produced in heavy-ion collisions. This suggests that the state of quarkonium will be substantially modified but perhaps not fully thermalized. Note also that, according to Fig. 7, the energy dependence reduces the rate by about a factor of 3 .

\section{B. A simplified model of quarkonium evolution}

We now move away from the static limit and consider a more "realistic" model for the quarkonium. This is based on the following assumptions:

(1) We neglect the quark-antiquark interaction in the octet channel, i.e., we set $V_{\mathrm{o}}=0$. This approximation would be justified in a large- $N_{c}$ limit, and it was used in the original derivation of the gluon-dissociation cross section [18]. Comparison with later derivations shows that it remains a reasonable approximation for $N_{c}=3[19,20]$.

(2) This implies in particular that the heavy particles behave as free particles once they are in the octet channel, and octet-to-octet transitions can be treated in the high-temperature limit. In this case, the corresponding equation of motion for the heavy quarks will reduce to a Langevin-type equation.

(3) We assume that a single bound state exists in the singlet channel. The survival probability of this singlet bound state is entirely controlled by its interaction with octet (continuum) states. In fact, we also ignore continuum singlet states (these represent only about $10 \%$ of the available continuum states).

In summary the model that we consider consists in one bound state, the singlet state, and a continuum of free, octet, states. We want to see how our equations describe the approach to equilibrium of this particular system.

We start with elementary remarks concerning the system when it is in thermal equilibrium with the plasma. Recall that we ignore the center-of-mass motion. We write the partition function of the relative motion as follows:

$$
\begin{aligned}
Z & =\mathrm{e}^{\left|E_{\mathrm{s}}\right| / T}+\Omega\left(N_{c}^{2}-1\right) \int_{p} \mathrm{e}^{-\frac{p^{2}}{M T}} \\
& =\mathrm{e}^{\left|E_{\mathrm{s}}\right| / T}+\frac{\Omega\left(N_{c}^{2}-1\right)}{\lambda_{T}^{3}}
\end{aligned}
$$

where $\left|E_{\mathrm{s}}\right|$ is the binding energy $\left(E_{\mathrm{s}}<0\right), \Omega$ is the volume of the plasma, and $\lambda_{T}=\sqrt{4 \pi / M T}$ is the thermal wavelength of the relative motion. At low temperature, the bound state dominates, and $Z \approx \mathrm{e}^{\left|E_{\mathrm{s}}\right| / T}$, while at high temperature $Z \approx \Omega\left(N_{c}^{2}-1\right) / \lambda_{T}^{3}$. Clearly, there is a transition temperature $T_{c}$, of the order of $\left|E_{\mathrm{s}}\right|$, corresponding to the situation where these two contributions are of the same order of magnitude,

$$
\mathrm{e}^{\left|E_{\mathrm{s}}\right| / T_{c}}=\frac{\Omega\left(N_{c}^{2}-1\right)}{\lambda_{T_{c}}^{3}} .
$$

Note that $T_{c}$ has a (weak, logarithmic) dependence on the volume, and would vanish in an infinite volume. Let $p^{\mathrm{s}}$ and $p^{\mathrm{o}}$ be the probabilities for the system to be in the ground state or in a continuum state, respectively. We have

$$
p_{\mathrm{eq}}^{\mathrm{s}}=\frac{\mathrm{e}^{\left|E_{\mathrm{s}}\right| / T}}{Z}, \quad p_{\mathrm{eq}}^{\mathrm{o}}=\frac{\Omega\left(N_{c}^{2}-1\right) / \lambda_{T}^{3}}{Z} .
$$

Clearly, when $T \ll T_{c}, p^{\mathrm{s}} \approx 1$, while $p^{\mathrm{o}} \approx 1$ when $T \gg T_{c}$.

The time evolution of the probabilities are given by the simplified system of equations

$$
\begin{aligned}
\frac{\mathrm{d} p^{\mathrm{s}}}{\mathrm{d} t}= & g^{2} C_{F} \int_{\boldsymbol{p}}\left(p_{\boldsymbol{p}}^{\mathrm{o}}-p^{\mathrm{s}} \mathrm{e}^{-\frac{E_{p}^{\mathrm{o}}-E^{\mathrm{s}}}{T}}\right) \\
& \times \int_{\boldsymbol{q}} \Delta^{>}\left(\omega_{\boldsymbol{p}}^{\mathrm{o}}-E^{\mathrm{s}}, \boldsymbol{q}\right)\left|\left\langle\mathrm{s}\left|\mathcal{S}_{\boldsymbol{q} \cdot \hat{\boldsymbol{r}}}\right| \mathrm{o}, \boldsymbol{p}\right\rangle\right|^{2},
\end{aligned}
$$

and

$$
\begin{aligned}
\frac{\partial p_{\boldsymbol{p}}^{\mathrm{o}}}{\partial t}- & \gamma \boldsymbol{\nabla}_{p}\left(\mathbf{p} p_{\boldsymbol{p}}^{\mathrm{o}}\right)-\frac{T \gamma M}{2} \Delta_{p} p_{\boldsymbol{p}}^{\mathrm{o}} \\
= & -\frac{g^{2}}{2 N_{c}} \frac{1}{\boldsymbol{\Omega}}\left(p_{\boldsymbol{p}}^{\mathrm{o}}-p^{\mathrm{s}} \mathrm{e}^{-\frac{E_{p}^{\mathrm{o}}-E^{\mathrm{s}}}{T}}\right) \\
& \times \int_{\boldsymbol{q}} \Delta^{>}\left(\omega_{\boldsymbol{p}}^{\mathrm{o}}-E^{\mathrm{s}}, \boldsymbol{q}\right)\left|\left\langle\mathrm{s}\left|\mathcal{S}_{\boldsymbol{q} \cdot \hat{r}}\right| \mathrm{o}, \boldsymbol{p}\right\rangle\right|^{2},
\end{aligned}
$$

where $E_{p}^{o}=p^{2} / M$ is the kinetic energy of the relative motion. We assume that the medium is contained in a cubic box of volume $\Omega$. Computations are made for two different volumes: $\Omega=1 \mathrm{fm}^{3}$ and $\Omega=100 \mathrm{fm}^{3}$ (these values cover the orders of magnitude of the typical volumes of the fireballs produced in a heavy-ion collision). This volume factor affects the numerical results, and it has been made explicit in Eq. (5.35). Thus, the plane wave in the equation above is normalized so that $\langle\mathbf{r} \mid \mathbf{o}, \boldsymbol{p}\rangle=\mathrm{e}^{i \mathbf{r} \cdot \mathbf{p}}$. 
TABLE I. Table showing different parameters that are used in our simulation at two different temperatures. Note that at $T=0$, i.e., in the vacuum, the binding energy of the singlet state is $E^{\mathrm{s}}=-372 \mathrm{MeV}$. The last column gives the decay width, $\Gamma_{\mathrm{s} \rightarrow \mathrm{o}}=a_{0}^{3} \int \mathrm{d}^{3} \boldsymbol{p} f\left(p a_{0}\right)$, with $f$ given in Eq. (5.38). The values in parentheses are obtained by using for the evaluation of $f\left(p a_{0}\right)$ the vacuum singlet bound-state energy and wave function.

\begin{tabular}{lcccc}
\hline \hline$T(\mathrm{MeV})$ & $E^{\mathrm{s}}(\mathrm{MeV})$ & $m_{D}(\mathrm{MeV})$ & $\alpha_{s}(2 \pi T)$ & $\Gamma_{\mathrm{s} \rightarrow \mathrm{o}}(\mathrm{MeV})$ \\
\hline 200 & -138.36 & 570.95 & 0.432 & $6.2(1.9)$ \\
400 & -51.57 & 955.15 & 0.302 & $39(13)$ \\
\hline \hline
\end{tabular}

The first equation expresses the change in the boundstate population, with a loss term caused by the singletto-octet transitions, while the gain term represents the possible transitions of any of the continuum octet states to the bound singlet. The second equation accounts in addition for the Brownian motion of the particles in the continuum. The specific form of the Langevin terms on the left-hand side is taken from Ref. [13]. As a simple consistency check of these equations, one may verify that

$$
\frac{\mathrm{d}}{\mathrm{d} t}\left(p^{\mathrm{s}}+\Omega\left(N_{c}^{2}-1\right) \int_{p} p_{p}^{\mathrm{o}}\right)=0
$$

and that the steady-state solution is given by Eq. (5.33).

We have solved Eqs. (5.34) and (5.35), taking for $\gamma$ the value used in Ref. [14], but adapted to the bottomonium mass, assuming that $\gamma$ goes as the inverse of the mass, that is, $\gamma=0.060 \mathrm{fm}^{-1}$. Other needed inputs are the binding energy and the wave function of the singlet ground state (that enters the computation of the matrix element $\left.\left\langle\mathrm{s}\left|\mathcal{S}_{q . \hat{r}}\right| \mathrm{o}, \boldsymbol{p}\right\rangle\right)$. We obtain these by solving the Schrödinger equation with a screened potential ${ }^{10}$

$$
V_{\mathrm{s}}=-C_{F} \alpha_{s}\left(1 / a_{0}\right) \frac{e^{-m_{D} r}}{r} .
$$

The results are shown in Table I. As can be seen in this table, screening substantially reduces the binding energy. Note that this reduction of the binding energy, together with the corresponding modification of the singlet wave function, entail a substantial increase of the decay width at a given temperature. This is of course in line with the energy dependence of the rates that we analyzed in the previous section. At $T=400 \mathrm{MeV}$, the decay width is of the same magnitude as the binding energy, suggesting that at this temperature the singlet can hardly be considered as a bound state anymore.

\footnotetext{
${ }^{10}$ This is done with the algorithm of Ref. [22], using its PYTHON implementation by Hector Martinez (https://github.com/ heedmane/schroepy).
}
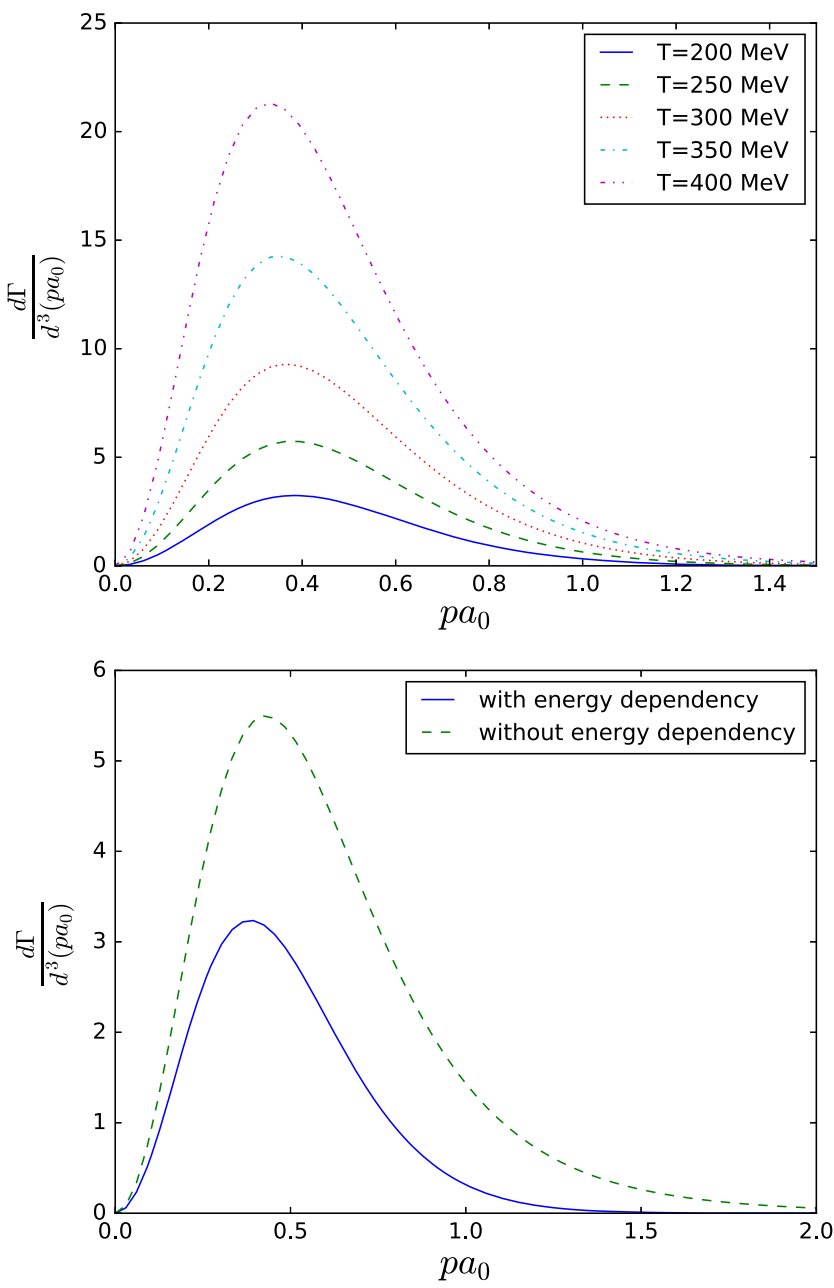

FIG. 8. Differential decay width of the singlet bound state into octets as a function of the octet momentum $p$, expressed in units of the inverse of the Bohr radius $a_{0}$ of the singlet. The top panel illustrates the dependence on the temperature. The bottom panel illustrates the effect of the energy dependence, at temperature $T=200 \mathrm{MeV}$ [the curve labeled "without energy dependence" corresponds to the function $f\left(p a_{0}\right)$ calculated by substituting $\left.\frac{p^{2}}{M}-E^{s} \rightarrow 0\right]$.

It is useful to introduce the following function, proportional to the differential decay rate of a singlet into an octet with momentum $\boldsymbol{p}$ :

$f\left(p a_{0}\right)=\frac{g^{2} C_{F}}{\left(2 \pi a_{0}\right)^{3}} \mathrm{e}^{-\frac{p^{2}}{M} E^{\mathrm{s}}} \int_{\boldsymbol{q}} \Delta^{>}\left(\frac{p^{2}}{M}-E^{\mathrm{s}}, \mathbf{q}\right)\left|\left\langle\mathrm{s}\left|S_{q}\right| \mathbf{o}, \boldsymbol{p}\right\rangle\right|^{2}$.

This function can be computed numerically once the singlet wave function is known. We use the HTL approximation to evaluate $\Delta^{>}(\omega, \mathbf{q})$ (see formulas in the previous subsection). The result of this computation at different temperatures is shown in Fig. 8. As one can see, the singlet state decays preferentially into octets whose momentum is of the 
TABLE II. $\quad p^{s}$ as obtained by solving Eqs. (5.39) and (5.40).

\begin{tabular}{ccccccc}
\hline \hline & \multicolumn{3}{c}{$\Omega=1 \mathrm{fm}^{3}$} & \multicolumn{4}{c}{$\Omega=100 \mathrm{fm}^{3}$} \\
\cline { 2 - 7 } & $5 \mathrm{fm} / \mathrm{c} 100 \mathrm{fm} / \mathrm{c}$ & Eq. & $5 \mathrm{fm} / \mathrm{c} 100 \mathrm{fm} / \mathrm{c}$ & Eq. \\
\hline$T=200 \mathrm{MeV}$ & 0.86 & 0.136 & 0.0814 & 0.85 & 0.0438 & 0.00089 \\
$T=400 \mathrm{MeV}$ & 0.39 & 0.0515 & 0.0175 & 0.36 & 0.0002 & 0.00018 \\
\hline \hline
\end{tabular}

order of half the inverse of the Bohr radius. We can rewrite the evolution equations of $p^{\mathrm{s}}$ and $p_{p}^{\mathrm{o}}$ in terms of this function $f$ :

$$
\frac{\mathrm{d} p^{\mathrm{s}}}{\mathrm{d} t}=a_{0}^{3} \int \mathrm{d}^{3} p\left(e^{\frac{p^{2}}{M^{-} E_{1 S}^{s}}} p_{\boldsymbol{p}}^{\mathrm{o}}-p^{\mathrm{s}}\right) f\left(p a_{0}\right),
$$

and

$$
\begin{aligned}
\frac{\mathrm{d} p_{\boldsymbol{p}}^{\mathrm{o}}}{\mathrm{d} t} & -\gamma \boldsymbol{\nabla}\left(\boldsymbol{p} p_{\boldsymbol{p}}^{\mathrm{o}}\right)-\frac{T \gamma M}{2} \Delta^{2} p_{\boldsymbol{p}}^{\mathrm{o}} \\
& =-\frac{\left(2 \pi a_{0}\right)^{3}}{\left(N_{c}^{2}-1\right) \Omega}\left(e^{\frac{p^{2}}{T} E_{1 S}^{\mathrm{s}}} p_{\boldsymbol{p}}^{\mathrm{o}}-p^{\mathrm{s}}\right) f\left(p a_{0}\right) .
\end{aligned}
$$

To solve these equations we use the same numerical methods as in Sec. VD of Ref. [13]. The most relevant difference compared to the case treated in Ref. [13] is that, in the present case, the singlet bound state can decay into octets with different momenta. To include this feature in our

TABLE III. $\quad p^{s}$ as obtained by solving Eqs. (5.39) and (5.40) without the Langevin-like term.

\begin{tabular}{ccccccc}
\hline \hline & \multicolumn{3}{c}{$\Omega=1 \mathrm{fm}^{3}$} & \multicolumn{4}{c}{$\Omega=100 \mathrm{fm}^{3}$} \\
\cline { 2 - 7 } & $5 \mathrm{fm} / \mathrm{c} 100 \mathrm{fm} / \mathrm{c}$ & Eq. & $5 \mathrm{fm} / \mathrm{c} 100 \mathrm{fm} / \mathrm{c}$ & Eq. \\
\hline$T=200 \mathrm{MeV}$ & 0.85 & 0.124 & 0.0814 & 0.85 & 0.0442 & 0.00089 \\
$T=400 \mathrm{MeV}$ & 0.40 & 0.0224 & 0.0175 & 0.38 & 0.001 & 0.00018 \\
\hline \hline
\end{tabular}

TABLE IV. $\quad p^{s}$ as obtained by solving Eqs. (5.39) and (5.40) ignoring the energy dependence of the imaginary part.

\begin{tabular}{cccccc}
\hline \hline & \multicolumn{2}{c}{$\Omega=1 \mathrm{fm}^{3}$} & & \multicolumn{2}{c}{$\Omega=100 \mathrm{fm}^{3}$} \\
\cline { 2 - 3 } \cline { 5 - 6 } & $5 \mathrm{fm} / \mathrm{c}$ & $100 \mathrm{fm} / \mathrm{c}$ & & $5 \mathrm{fm} / \mathrm{c}$ & $100 \mathrm{fm} / \mathrm{c}$ \\
\hline$T=200 \mathrm{MeV}$ & 0.5631 & 0.0093 & & 0.5596 & 0.001 \\
\hline \hline
\end{tabular}

simulation, we use a rejection sampling based on the differential decay width to select the momentum.

The value of the survival probability of the singlet state, $p^{\mathrm{s}}$, obtained by solving these equations is given in Table II, for two different temperatures, two different interaction times, and two different volumes. For comparison the value of the equilibrium probability is indicated in the last column.

We see that at late times $p^{\mathrm{s}}$ becomes very small: the system is then completely dominated by the octets, the more so the larger the volume. However, on time scales that are typical of the lifetime of the plasma in a heavy-ion collision, $\sim 5 \mathrm{fm} / \mathrm{c}$ a significant amount of singlets survive, with the survival probability being essentially independent of the volume. The reason for this is that the lifetime of the singlet is independent of the volume while that of the octets (which does depend on the volume) is much bigger than $5 \mathrm{fm} / \mathrm{c}$ whenever $\Omega \gg a_{0}^{3}$ (as it happens in heavy-ion collisions).

In order to get a feeling for the role of the Brownian motion of the free quarks, we have repeated the calculations dropping the Langevin terms on the left-hand side of Eq. (5.40), i.e., keeping only the time derivative. We obtain then the results listed in Table III. By comparing with Table II, one sees that Brownian motion does not affect much $p_{s}$ at small times. However it produces a momentum broadening which, for small volumes, slows down the disappearance of the singlet bound state (i.e., delays the approach to equilibrium), as can be seen by comparing the results obtained at $100 \mathrm{fm} / \mathrm{c}$ and $\Omega=1 \mathrm{fm}$ with (Table II) and without (Table III) Brownian motion. Note that this effect is negligible in large volumes $(\Omega=100 \mathrm{fm})$; this is because in large volumes, the decay of octets into singlets is negligible.

We have also repeated the calculation, ignoring the energy dependence of the imaginary potential [that is, we set $\mathrm{e}^{\frac{p^{2}-E_{1 S}^{s}}{T}}=1$ and use $\Delta^{>}(0, \mathbf{q})$ in Eq. (5.38)]. The results are given in Table IV, for the case $T=200 \mathrm{MeV}$. As can be seen from this table, the survival probability is greatly reduced, and it eventually vanishes at large time: the absence of an energetic penalty for the transition to an octet state allows for a rapid occupation of the large continuum phase space. This provides another indication of the importance of the energy dependence of the rates.

As a final remark, we have evaluated the free energy, internal energy and the entropy in equilibrium. These are

\begin{tabular}{|c|c|c|c|c|c|c|}
\hline & \multicolumn{3}{|c|}{$\Omega=1 \mathrm{fm}^{3}$} & \multicolumn{3}{|c|}{$\Omega=100 \mathrm{fm}^{3}$} \\
\hline & $F \mathrm{MeV}$ & $E \mathrm{MeV}$ & $T S \mathrm{MeV}$ & $F \mathrm{MeV}$ & $E \mathrm{MeV}$ & $T S \mathrm{MeV}$ \\
\hline$T=200 \mathrm{MeV}$ & -640.07 & 264.32 & 904.39 & -1544.30 & 299.61 & 1843.91 \\
\hline$T=400 \mathrm{MeV}$ & -1669.14 & 588.58 & 2257.72 & -3504.21 & 599.88 & 4104.09 \\
\hline
\end{tabular}
shown in Table V. In all cases, the free energy is dominated

TABLE V. Thermodynamic potentials at equilibrium for the discussed model. 
by the entropy, the more so the larger $T$ and/or $\Omega$. Note that in the large-volume $\Omega$ limit the thermal energy $E$ goes to a constant, while $F \sim-T S$ goes to infinity but just as $-T \log \Omega$.

\section{CONCLUSIONS}

The equations for the reduced density matrix that we have derived in this paper describe the evolution of a quarkonium towards thermal equilibrium in both regimes of high and moderate temperatures. The high-temperature regime is that where the binding energies can be ignored. Then the dynamics is well described by a Lindblad equation and the approach to equilibrium is controlled by the increase of the von Neumann entropy. In this regime, binding forces can be treated perturbatively, and the effect of the collisions is accounted for by a static imaginary potential. In the regime of moderate temperature, binding energies cannot be ignored, and it is convenient to use as a reference basis, that of the eigenstates of the effective heavy-quark Hamiltonian. The equation for the density matrix then leads to rate equations describing transitions between these eigenstates, and the approach to equilibrium is accompanied by the decrease of the free energy. The dynamics of continuum states remains dominated by Brownian motion and is described by a Langevin equation. In this regime of moderate temperature, the effect of collisions is still captured by an imaginary potential, which enters the determination of the collision rates. An important feature of the imaginary potential is that it depends on the energy: this is because as the energy of a transition increases, the phase space of the space-like gluons that cause this transition decreases. This energy dependence is found to be numerically important and should be taken into account in phenomenological studies. As we have emphasized, this effect is expected to be much more important for QCD than it would be for QED. This is because the absorption of a gluon by a quark-antiquark pair changes the color state of the pair, and turns an attractive force into a repulsive one, or vice versa.

The last section of the paper presented numerical studies illustrating the main concepts discussed in the earlier sections. The first example is that of an infinitely massive quark-antiquark pair. This is close to the typical setup used in lattice gauge calculations, and some comparison with recent lattice results has been attempted. It would certainly be worthwhile to extend such comparison and see whether the strong suppression arising from the energy dependence of the imaginary part of the potential at small separation can be reproduced by lattice calculations. The second example considered a simplified model of a quarkonium with a single bound state in a singlet state, and continuum octet states. Although this is an oversimplification of the realistic situation, many interesting features emerge from this study, that could be relevant in phenomenological studies. This example illustrates in particular the interplay between screening and collisions, and the importance of treating both on the same footing, as we did in this paper. ${ }^{11}$

In this paper we have focused on a simple question, namely, how a quarkonium approaches equilibrium when it is in contact with a static quark-gluon plasma in thermal equilibrium at temperature $T$. Although we have examined only simplified models, the equations that we have derived allow in principle for a quantitative answer to this question. They should provide a consistent starting point for more elaborate phenomenological work. The formalism developed in this paper should be well suited to the study of the bottomonium, presumably to be found in the moderatetemperature regime in heavy-ion collisions. The case of charmonium is more intricate, and presumably calls for a mix of techniques, in particular if one wishes to address the issue of recombination. Then the approximations developed in Ref. [13] may be useful.

\section{ACKNOWLEDGMENTS}

This work has been supported in part by the European Research Council under the Advanced Investigator Grant No. ERC-AD-267258. The work of M. A. E. was supported by the Academy of Finland, Project No. 303756.

\section{APPENDIX A: ELIMINATION OF THE CENTER-OF-MASS COORDINATES}

In this Appendix, we perform the Fourier transform of our main equation, and eliminate the center-of-mass coordinate.

In order to proceed with the Fourier transform, we note that the correlator $\Delta_{-}^{<}\left(X, X^{\prime}\right)$ defined in Eq. (2.30) depends on the difference of times, $\tau=t-t^{\prime}$, and a priori on four coordinates. Because of translation invariance, it is in fact a function of only three differences of coordinates. To make this more explicit, we consider $\Delta_{-}^{\leq}\left(X, X^{\prime}\right)$ as an operator in the two-particle space, with matrix elements

$$
\begin{aligned}
\left\langle\boldsymbol{r}_{1}, \overline{\boldsymbol{r}}_{1}\left|\Delta_{-}^{<}\left(\tau, \boldsymbol{X}, \boldsymbol{X}^{\prime}\right)\right| \boldsymbol{r}_{2}, \overline{\boldsymbol{r}}_{2}\right\rangle & \\
= & \Delta^{<}(\boldsymbol{Y}+\boldsymbol{y} / 2)+\Delta^{<}(\boldsymbol{Y}-\boldsymbol{y} / 2)-\Delta^{<}(\boldsymbol{Y}+\boldsymbol{r}) \\
& -\Delta^{<}(\boldsymbol{Y}-\boldsymbol{r}) \\
\equiv & \Delta_{-}(\tau ; \boldsymbol{Y}, \boldsymbol{y}, \boldsymbol{r}),
\end{aligned}
$$

where

$$
\begin{aligned}
& \boldsymbol{R}_{i} \equiv \frac{\boldsymbol{r}_{i}+\overline{\boldsymbol{r}}_{i}}{2}, \quad \boldsymbol{s}_{i} \equiv \boldsymbol{r}_{i}-\overline{\boldsymbol{r}}_{i}, \quad(i=1,2) \\
& \boldsymbol{Y} \equiv \boldsymbol{R}_{1}-\boldsymbol{R}_{2}, \quad \boldsymbol{y} \equiv \boldsymbol{s}_{1}-\boldsymbol{s}_{2}, \quad \boldsymbol{r} \equiv \frac{\boldsymbol{s}_{1}+\boldsymbol{s}_{2}}{2} .
\end{aligned}
$$

\footnotetext{
${ }^{11}$ The effect of the change in the binding energies on the collision rates is taken into account in some recent phenomenological analyses, such as that based on the in-medium $T$ matrix [23].
} 
The coordinates $\boldsymbol{Y}$ and $\boldsymbol{y}$ play an important role in the semiclassical approximation (see Ref. [13]). Thus, in the large-mass limit, $\boldsymbol{r}_{1} \approx \boldsymbol{r}_{2}$ and $\overline{\boldsymbol{r}}_{1} \approx \overline{\boldsymbol{r}}_{2}, \boldsymbol{Y} \rightarrow 0, \boldsymbol{y} \rightarrow 0$ and $\boldsymbol{r}$ becomes equal to the relative coordinate. It is convenient to express the Fourier transforms in terms of these variables. We have, for instance (with the shorthand notation $\left.\int_{\boldsymbol{q}}=\int \frac{\mathrm{d}^{3} \boldsymbol{q}}{(2 \pi)^{3}}\right)$

$$
\begin{aligned}
\Delta^{<}\left(\tau, \boldsymbol{r}_{1}-\boldsymbol{r}_{2}\right) & =\int_{\boldsymbol{q}} \mathrm{e}^{i \boldsymbol{q} \cdot\left(\boldsymbol{r}_{1}-\boldsymbol{r}_{2}\right)} \Delta^{<}(\tau, \boldsymbol{q}) \\
& =\int_{\boldsymbol{q}} \mathrm{e}^{i \boldsymbol{q} \cdot \boldsymbol{Y}} \mathrm{e}^{i \boldsymbol{q} \cdot \boldsymbol{y} / 2} \Delta^{<}(\tau, \boldsymbol{q}),
\end{aligned}
$$

and for $\Delta_{-}^{<}(\tau ; \boldsymbol{Y}, \boldsymbol{y}, \boldsymbol{r})$,

$\Delta_{-}(\tau ; \boldsymbol{Y}, \boldsymbol{y}, \boldsymbol{r})=2 \int_{\boldsymbol{q}} \mathrm{e}^{i \boldsymbol{q} \cdot \boldsymbol{Y}}[\cos (\boldsymbol{q} \cdot \boldsymbol{y} / 2)-\cos (\boldsymbol{q} \cdot \boldsymbol{r})] \Delta(\tau, \boldsymbol{q})$.

The variable $\boldsymbol{q}$ represents the momentum of the exchange gluon. We can also take a Fourier transform with respect to the time variable $\left(q=\left(q_{0}, \boldsymbol{q}\right)\right)$

$$
\Delta(\tau, \boldsymbol{q})=\int_{q_{0}} \mathrm{e}^{-i q_{0} \tau} \Delta(q)
$$

and write (now with $\int_{q}=\int \frac{\mathrm{d}^{4} q}{(2 \pi)^{4}}$ )

$$
\Delta_{-}(\tau ; \boldsymbol{Y}, \boldsymbol{y}, \boldsymbol{r})=2 \int_{q} \Delta_{-}(q, \boldsymbol{y}, \boldsymbol{r}) \mathrm{e}^{-i q_{0} \tau} \mathrm{e}^{i \boldsymbol{q} \cdot \boldsymbol{Y}} .
$$

We shall sometimes write, with a slight abuse of notation, $\Delta_{-}\left(q, \boldsymbol{s}_{1}, \boldsymbol{s}_{2}\right)$ in place of $\Delta_{-}(q, \boldsymbol{y}, \boldsymbol{r})$. To summarize, we can write $\left\langle\boldsymbol{r}_{1}, \overline{\boldsymbol{r}}_{1}\left|\Delta_{ \pm}^{<}\left(\tau, \boldsymbol{X}, \boldsymbol{X}^{\prime}\right)\right| \boldsymbol{r}_{2}, \overline{\boldsymbol{r}}_{2}\right\rangle$ as

$$
\Delta_{ \pm}^{<}\left(\boldsymbol{R}_{1}, \boldsymbol{R}_{2}, \boldsymbol{s}_{1}, \boldsymbol{s}_{2} ; \tau\right)=2 \int \frac{\mathrm{d}^{4} q}{(2 \pi)^{4}} \Delta_{ \pm}^{<}\left(q, \boldsymbol{s}_{1}, \boldsymbol{s}_{2}\right) \mathrm{e}^{-i q_{0} \tau+i \boldsymbol{q} \cdot\left(\boldsymbol{R}_{1}-\boldsymbol{R}_{2}\right)},
$$

where $q=\left(q_{0}, \mathbf{q}\right)$ represents the exchanged gluon fourmomentum, and

$$
\begin{aligned}
& \Delta_{ \pm}^{<}\left(q, \boldsymbol{s}_{1}, \boldsymbol{s}_{2}\right) \\
& \quad=\Delta^{<}(q)\left[\cos \left(\frac{\mathbf{q}\left(\boldsymbol{s}_{1}-\boldsymbol{s}_{2}\right)}{2}\right) \pm \cos \left(\frac{\mathbf{q}\left(\boldsymbol{s}_{1}+\boldsymbol{s}_{2}\right)}{2}\right)\right] .
\end{aligned}
$$

Similar relations hold for $\Delta_{ \pm}^{>}\left(\boldsymbol{R}_{1}, \boldsymbol{R}_{2}, \boldsymbol{s}_{1}, \boldsymbol{s}_{2} ; \tau\right)$.

These relations allow us to perform the partial trace over the center-of-mass coordinate, or equivalently over the center-of-mass momentum. We illustrate the procedure with the first term of Eq. (2.23), and more precisely the first contribution to Eq. (2.28). Consider then the matrix element

$$
\left\langle\boldsymbol{R}_{1}, \boldsymbol{s}_{1}\left|\mathcal{P}_{X} U_{\mathrm{o}}(\tau) \mathcal{P}_{X^{\prime}}(\tau) D_{\mathrm{s}}(t-\tau) U_{\mathrm{s}}^{\dagger}(\tau)\right| \boldsymbol{R}_{2}, \boldsymbol{s}_{2}\right\rangle \Delta_{-}^{>}\left(X, X^{\prime}\right)
$$

between localized states of the heavy quark-antiquark pair (in a color-singlet state). Taking advantage of the fact that the projectors are diagonal in coordinate space, we can rewrite this as (omitting time variables to simplify the writing)

$$
\int_{\boldsymbol{X}_{2}^{\prime}, \overline{\boldsymbol{X}}_{2}}\left\langle\boldsymbol{X}_{1}\left|U_{\mathrm{o}}\right| \boldsymbol{X}_{2}^{\prime}\right\rangle\left\langle\boldsymbol{X}_{2}^{\prime}\left|D_{\mathrm{s}}\right| \overline{\boldsymbol{X}}_{2}\right\rangle\left\langle\overline{\boldsymbol{X}}_{2}\left|U_{\mathrm{s}}^{\dagger}\right| \boldsymbol{X}_{2}\right\rangle \Delta_{-}^{>}\left(\tau, \boldsymbol{X}_{1}, \boldsymbol{X}_{2}^{\prime}\right)
$$

where $\boldsymbol{X}_{i}=\left(\boldsymbol{R}_{i}, \boldsymbol{s}_{i}\right)$. We note then that the evolution operators factorize into a center-of-mass contribution which depends only on the kinetic energy of the center of mass, and a part related to the relative motion that involves the potentials $V_{\mathrm{s}}$ or $V_{\mathrm{o}}$. We set $U_{\mathrm{s}}=U_{\mathrm{cm}} \tilde{U}_{\mathrm{s}}$ and similarly for $U_{\mathrm{o}}$. We have

$$
\begin{aligned}
\left\langle\boldsymbol{X}_{1}\left|U_{\mathrm{o}}(\tau)\right| \boldsymbol{X}_{2}^{\prime}\right\rangle & =\left\langle\boldsymbol{R}_{1}, \boldsymbol{s}_{1}\left|U_{\mathrm{cm}} \tilde{U}_{\mathrm{o}}\right| \boldsymbol{R}_{2}^{\prime}, \boldsymbol{s}_{2}^{\prime}\right\rangle \\
& =\left\langle\boldsymbol{R}_{1}\left|U_{\mathrm{cm}}\right| \boldsymbol{R}_{2}^{\prime}\right\rangle\left\langle\boldsymbol{s}_{1}\left|\tilde{U}_{\mathrm{o}}\right| \boldsymbol{s}_{2}^{\prime}\right\rangle,
\end{aligned}
$$

where

$$
\left\langle\boldsymbol{R}_{1}\left|U_{\mathrm{cm}}\right| \boldsymbol{R}_{2}^{\prime}\right\rangle=\int_{\boldsymbol{P}_{1}^{\prime}} \mathrm{e}^{-i \tau_{\frac{1}{4 M}}^{P^{\prime 2}}} \mathrm{e}^{i \boldsymbol{P}_{1}^{\prime} \cdot\left(\boldsymbol{R}_{1}-\boldsymbol{R}_{2}^{\prime}\right)},
$$

and $\tilde{U}_{\mathrm{o}}$ acts on the relative coordinates, in the octet channel. A simple calculation then yields for the matrix element of Eq. (A9) between center-of-mass momentum states $P_{1}$ and $P_{2}$,

$$
\begin{aligned}
& \int_{\boldsymbol{s}_{2}^{\prime}, \boldsymbol{s}_{2}^{\prime \prime}}\left\langle\boldsymbol{s}_{1}\left|\tilde{U}_{\mathrm{o}}\right| \boldsymbol{s}_{2}^{\prime}\right\rangle\left\langle\boldsymbol{P}_{1}, \boldsymbol{s}_{2}^{\prime}\left|D_{\mathrm{s}}\right| \boldsymbol{P}_{2}, \boldsymbol{s}_{2}^{\prime \prime}\right\rangle\left\langle\boldsymbol{s}_{2}^{\prime \prime}\left|\tilde{U}_{\mathrm{s}}^{\dagger}\right| \boldsymbol{s}_{2}\right\rangle \\
& \times \Delta_{-}^{\geq}\left(\tau ; \boldsymbol{q}, \boldsymbol{s}_{1}, \boldsymbol{s}_{2}^{\prime}\right) \mathrm{e}^{-i \tau \frac{\left(\boldsymbol{P}_{1}-q\right)^{2}}{4 M}} \mathrm{e}^{i \tau_{2}^{2}}
\end{aligned}
$$

which can also be written as an operator equation in the space of relative coordinates

$$
\begin{gathered}
\int_{\boldsymbol{s}^{\prime}} \mathcal{P}_{\boldsymbol{s}} \tilde{U}_{\mathrm{o}}(\tau) \mathcal{P}_{\boldsymbol{s}^{\prime}}\left\langle\boldsymbol{P}_{1}\left|D_{\mathrm{s}}(t-\tau)\right| \boldsymbol{P}_{2}\right\rangle \tilde{U}_{\mathrm{s}}^{\dagger}(\tau) \\
\times \Delta_{-}^{\geq}\left(\tau ; \boldsymbol{q}, \boldsymbol{s}, \boldsymbol{s}^{\prime}\right) \mathrm{e}^{-i \tau \frac{\left(\boldsymbol{P}_{1}-q\right)^{2}-P_{2}^{2}}{4 M}} .
\end{gathered}
$$

At this point we recall that

$$
\Delta_{-}^{>}\left(q, \boldsymbol{s}, \boldsymbol{s}^{\prime}\right)=2 \sin \frac{\boldsymbol{q} \cdot \boldsymbol{s}}{2} \sin \frac{\boldsymbol{q} \cdot \boldsymbol{s}^{\prime}}{2} \Delta^{>}(q),
$$


so that the first contribution to $\mathcal{L}^{\text {ss }}(\tau) D_{\mathrm{s}}(t-\tau)$ in Eq. (2.28) finally reads

$$
\begin{aligned}
- & g^{2} C_{F} \int_{0}^{t-t_{0}} \mathrm{~d} \tau \int_{q} \mathrm{e}^{-i q_{0} \tau} \Delta^{>}(q) \\
& \times \mathcal{S}_{q \cdot \hat{s}} \tilde{U}_{\mathrm{o}}(\tau) \mathcal{S}_{q \cdot \hat{s}} \tilde{U}_{\mathrm{s}}^{\dagger}(\tau)\left\langle\boldsymbol{P}_{1}\left|D_{\mathrm{s}}(t)\right| \boldsymbol{P}_{2}\right\rangle \mathrm{e}^{-i \tau \frac{\left(\boldsymbol{P}_{1}-q\right)^{2}-P_{2}^{2}}{4 M}}
\end{aligned}
$$

where

$$
\mathcal{S}_{\boldsymbol{q} \cdot \boldsymbol{s}} \equiv 2 \sin (\boldsymbol{q} \cdot \hat{\boldsymbol{s}} / 2)
$$

and $\hat{\boldsymbol{s}}$ is the relative coordinate operator.

At this point it is (almost) trivial to trace out the center-of-mass d.o.f. This amounts to setting $\boldsymbol{P}_{1}=\boldsymbol{P}_{2}$ and integrating over $\boldsymbol{P}_{1}$. Note that the commutator in Eq. (2.23) yields

$$
\begin{aligned}
& \left\langle\boldsymbol{P}_{1}, \boldsymbol{s}_{1}\left|\left[H_{Q}, D_{\mathrm{s}}\right]\right| \boldsymbol{P}_{2}, \boldsymbol{s}_{2}\right\rangle \\
& \quad=\frac{\boldsymbol{P}_{1}^{2}-\boldsymbol{P}_{2}^{2}}{4 M}\left\langle\boldsymbol{P}_{1}, \boldsymbol{s}_{1}\left|D_{\mathrm{s}}\right| \boldsymbol{P}_{2}, \boldsymbol{s}_{2}\right\rangle+\left\langle\boldsymbol{P}_{1}, \boldsymbol{s}_{1}\left|\left[H_{\mathrm{s}}, D_{\mathrm{s}}\right]\right| \boldsymbol{P}_{2}, \boldsymbol{s}_{2}\right\rangle .
\end{aligned}
$$

The first term will not contribute when taking the trace (with $\boldsymbol{P}_{1}=\boldsymbol{P}_{2}$ ). As for the second term, it yields $\left\langle\boldsymbol{s}_{1}\left|\left[H_{\mathrm{s}}, \tilde{D}_{\mathrm{s}}\right]\right| \boldsymbol{s}_{2}\right\rangle$.

To proceed further we need to analyze the phase factor in Eq. (A16). This is the product of $\tau$ and the recoil energy

$$
\Delta E_{\text {recoil }}=\frac{(\boldsymbol{P}-\boldsymbol{q})^{2}}{4 M}-\frac{\boldsymbol{P}^{2}}{4 M}=\frac{1}{4 M}\left(\boldsymbol{q}^{2}-2 \boldsymbol{P} \cdot \boldsymbol{q}\right)
$$

where we have set $P_{1}=P_{2}=P$. The quantity $\Delta E_{\text {recoil }}$ is the recoil energy of the heavy-quark system, after absorption or emission of a gluon with momentum $\boldsymbol{q}$. The range of the $\tau$ integration in Eq. (A16) is limited by the propagator $\Delta^{>}(\tau, \boldsymbol{q})$ to be of the order of the inverse Debye mass $m_{D} \lesssim T$. On the other hand, the collisions of the heavy particles with the light constituents of the plasma involve the exchange of soft gluons, with $|\boldsymbol{q}| \lesssim m_{D}$. It follows that typically, $q \tau \sim 1$, and the recoil energy is a small fraction of the Debye mass, $\tau q^{2} / M \lesssim\left(m_{D} / M\right)$. A similar estimate holds for the term $\tau \boldsymbol{P} \cdot \boldsymbol{q} / M \lesssim(T / M)$, where we have assumed that $P \lesssim T$. (We consider pairs that are initially at rest. If the center-of-mass momentum is high then we need to consider "hot wind" effects, which is beyond the scope of this paper [24,25].) Since we assume that both $m_{D} \ll M$ and $T \ll M$, one can safely ignore the phase factor.

A similar reasoning can be made for all the contributions to the main equations. We then obtain the equations that are listed in the main text.

\section{APPENDIX B: MULTIPLE-SCALE ANALYSIS}

In this Appendix, we discuss the solution of Eq. (3.28) within perturbation theory, paying particular attention to the secular terms. We first rewrite Eq. (3.25) as follows:

$$
\frac{\mathrm{d} \mathcal{D}}{d t}+i[H, \mathcal{D}]=\epsilon \mathcal{F}[\mathcal{D}],
$$

where $\mathcal{F}$ is a linear functional of $\mathcal{D}$ and $\epsilon$ is a small parameter. We regard the right-hand side as a perturbation and attempt to solve Eq. (B1) as an expansion in powers of $\epsilon$. That is, we write

$$
\mathcal{D}(t)=\mathcal{D}_{0}(t)+\epsilon \mathcal{D}_{1}(t)+\cdots
$$

and obtain

$$
\mathcal{D}_{0}(t)=e^{-i H t} \mathcal{D}_{0}(0) e^{i H t},
$$

and

$$
\mathcal{D}_{1}(t)=\int_{0}^{t} d t^{\prime} e^{-i H\left(t-t^{\prime}\right)} \mathcal{F}\left[\mathcal{D}_{0}\left(t^{\prime}\right)\right] e^{i H\left(t-t^{\prime}\right)} .
$$

The difficulty with this naive expansion is that the condition $\epsilon \mathcal{D}_{1} \ll \mathcal{D}_{0}$ is not always satisfied. In particular, this condition is violated at late times if the quantity

$\mathcal{F}_{S}\left[\mathcal{D}_{0}(t)\right]=\lim _{T \rightarrow \infty} \frac{1}{T} \int_{0}^{T} d t^{\prime} e^{-i H\left(t-t^{\prime}\right)} \mathcal{F}\left[\mathcal{D}_{0}\left(t^{\prime}\right)\right] e^{i H\left(t-t^{\prime}\right)}$,

is not equal to zero. Let us then set

$$
\mathcal{F}\left[\mathcal{D}_{0}(t)\right]=\mathcal{F}_{S}\left[\mathcal{D}_{0}(t)\right]+\delta \mathcal{F}\left[\mathcal{D}_{0}(t)\right]
$$

so that

$$
\begin{aligned}
\mathcal{D}_{1}(t)= & t e^{-i H t} \mathcal{F}_{S}\left[\mathcal{D}_{0}(0)\right] e^{i H t} \\
& +\int_{0}^{t} d t^{\prime} e^{-i H\left(t-t^{\prime}\right)} \delta \mathcal{F}\left[\mathcal{D}_{0}\left(t^{\prime}\right)\right] e^{i H\left(t-t^{\prime}\right)} .
\end{aligned}
$$

This expression makes explicit the secular term, growing linearly with time, at the origin of the breakdown of naive perturbation theory.

The problem can be handled by multiple-scale analysis (see e.g., Chapter 11 of Ref. [26]). One introduces a "slow" time $\tau=\epsilon t$, and considers $D$ as a function of $t$ and $\tau$, treated (artificially) as independent variables. We have, as before,

$$
\mathcal{D}(t, \tau)=\mathcal{D}_{0}(t, \tau)+\epsilon \mathcal{D}_{1}(t, \tau)+\cdots .
$$

The leading-order equation reads 


$$
\frac{\partial \mathcal{D}_{0}}{\partial t}+i\left[H, \mathcal{D}_{0}\right]=0
$$

so that

$$
\mathcal{D}_{0}(t, \tau)=e^{-i H t} \mathcal{D}_{0}(0, \tau) e^{i H t} .
$$

The next-to-leading-order equation involves the derivative of $\mathcal{D}_{0}(t, \tau)$ with respect to $\tau$, viz.

$$
\epsilon \frac{\partial \mathcal{D}_{0}}{\partial \tau}+\epsilon \frac{\partial \mathcal{D}_{1}}{\partial t}+i \epsilon\left[H, \mathcal{D}_{1}\right]=\epsilon \mathcal{F}\left[\mathcal{D}_{0}(t, \tau)\right]
$$

We can now use $\frac{\partial \mathcal{D}_{0}}{\partial \tau}$ in order to cancel the secular contribution of $\mathcal{F}\left[\mathcal{D}_{0}(t, \tau)\right]$, that is, we demand that the equation

$$
\frac{d \mathcal{D}_{0}}{d \tau}=\mathcal{F}_{S}\left[\mathcal{D}_{0}(t, \tau)\right]
$$

be satisfied. This fixes the $\tau$ dependence of $\mathcal{D}_{0}(t, \tau)$. Then we can solve for $\mathcal{D}_{1}$,

$$
\mathcal{D}_{1}(t, \tau)=\int_{0}^{t} d t^{\prime} e^{-i H\left(t-t^{\prime}\right)} \delta \mathcal{F}\left[\mathcal{D}_{0}\left(t^{\prime}, \tau\right)\right] e^{i H\left(t-t^{\prime}\right)} .
$$

By construction, $\mathcal{D}_{1}(t, \tau)$ no longer contains a secular term, and can be considered a genuine perturbative quantity. A similar result could have been obtained by applying renormalization-group techniques, as discussed recently in Ref. [27].

The separation of the secular term requires the solution of Eq. (B12). By projecting this equation on the eigenvectors of the operator $H$, assuming that all energy levels are discrete, we obtain

$$
\begin{aligned}
&\langle n| \mathcal{F}_{S} {\left[\mathcal{D}_{0}(t, \tau)\right]|m\rangle } \\
&= \lim _{T \rightarrow \infty} \frac{1}{T} \int_{0}^{T} d t^{\prime} \sum_{n^{\prime} m^{\prime}} e^{-i t\left(E_{n}-E_{m}\right)} e^{i t^{\prime}\left(E_{n}-E_{n^{\prime}}-E_{m}+E_{m^{\prime}}\right)} \\
& \quad \times\left\langle n\left|\mathcal{F}\left[\mathcal{P}_{n^{\prime}} \mathcal{D}_{0}(0, \tau) \mathcal{P}_{m^{\prime}}\right]\right| m\right\rangle
\end{aligned}
$$

This is nonzero only if $E_{n}-E_{m}=E_{n^{\prime}}-E_{m^{\prime}}$. Thus the evolution described by Eq. (B12) only connects pairs of states whose energy differences $E_{m n}=E_{m}-E_{n}$ are identical. It follows in particular that the evolution of the populations of the various quarkonium states, i.e., of the diagonal elements, for which $E_{m n}=0$, decouples from that of the nondiagonal ones (at leading order and assuming the absence of degenerate states). One can also similarly evaluate the matrix elements of $\mathcal{D}_{1}(t, \tau)$. One gets

$$
\begin{aligned}
\left\langle n\left|\mathcal{D}_{1}(t, \tau)\right| m\right\rangle= & -i \sum_{n^{\prime} m^{\prime}} \frac{\mathrm{e}^{-i t E_{n m}}-\mathrm{e}^{-i t E_{n^{\prime} m^{\prime}}}}{E_{n^{\prime} m^{\prime}}-E_{n m}} \\
& \times\left\langle n\left|\delta \mathcal{F}\left[\left\langle n^{\prime}\left|D_{0}(0, \tau)\right| m^{\prime}\right\rangle\right]\right| m\right\rangle,
\end{aligned}
$$

where, by construction, $E_{n^{\prime} m^{\prime}} \neq E_{n m}$. Assuming that the particles are confined in a volume $\Omega \sim L^{3}$, the lowest values of the energy denominators are of order $L^{-1}$. Thus, if $\Gamma$ denotes the leading-order decay rate, $\mathcal{D}_{1}$ will remain a small perturbative correction as long as $\Gamma L \ll 1$. In the example treated in Sec V B, this condition is well satisfied for $\Omega=1 \mathrm{fm}^{3}$, but only marginally for $\Omega=100 \mathrm{fm}^{3}$.
[1] E. Scomparin, Quarkonium production in $A A$ and $p A$ collisions, Nucl. Phys. A967, 208 (2017).

[2] M. Laine, O. Philipsen, P. Romatschke, and M. Tassler, Real-time static potential in hot QCD, J. High Energy Phys. 03 (2007) 054.

[3] A. Beraudo, J. P. Blaizot, and C. Ratti, Real and imaginarytime $Q \bar{Q}$ correlators in a thermal medium, Nucl. Phys. A806, 312 (2008).

[4] N. Brambilla, J. Ghiglieri, A. Vairo, and P. Petreczky, Static quark-antiquark pairs at finite temperature, Phys. Rev. D 78, 014017 (2008).

[5] M. A. Escobedo and J. Soto, Non-relativistic bound states at finite temperature (I): The Hydrogen atom, Phys. Rev. A 78, 032520 (2008).

[6] M. A. Escobedo and J. Soto, Non-relativistic bound states at finite temperature (II): The muonic hydrogen, Phys. Rev. A 82, 042506 (2010).
[7] A. Rothkopf, T. Hatsuda, and S. Sasaki, Complex HeavyQuark Potential at Finite Temperature from Lattice QCD, Phys. Rev. Lett. 108, 162001 (2012).

[8] Y. Burnier, O. Kaczmarek, and A. Rothkopf, Quarkonium at finite temperature: Towards realistic phenomenology from first principles, J. High Energy Phys. 12 (2015) 101.

[9] H. P. Breuer and F. Petruccione, The Theory of Open Quantum Systems (Oxford University, New York, 2002).

[10] Y. Akamatsu, Heavy quark master equations in the Lindblad form at high temperatures, Phys. Rev. D 91, 056002 (2015).

[11] A. Rothkopf, A first look at bottomonium melting via a stochastic potential, J. High Energy Phys. 04 (2014) 085.

[12] R. Katz and P. B. Gossiaux, The Schrödinger-Langevin equation with and without thermal fluctuations, Ann. Phys. (Amsterdam) 368, 267 (2016). 
[13] J.-P. Blaizot and M. A. Escobedo, Quantum and classical dynamics of heavy quarks in a quark-gluon plasma, J. High Energy Phys. 06 (2018) 034.

[14] J.-P. Blaizot, D. De Boni, P. Faccioli, and G. Garberoglio, Heavy quark bound states in a quarkgluon plasma: Dissociation and recombination, Nucl. Phys. A946, 49 (2016).

[15] G. Lindblad, On the generators of quantum dynamical semigroups, Commun. Math. Phys. 48, 119 (1976).

[16] F. Benatti and H. Narnhofer, Entropy behavior under completely positive maps, Lett. Math. Phys. 15, 325 (1988).

[17] H. Spohn, Entropy production for quantum dynamical semigroups, J. Math. Phys. 19, 1227 (1978).

[18] G. Bhanot and M. E. Peskin, Short distance analysis for heavy quark systems. 2. Applications, Nucl. Phys. B156, 391 (1979).

[19] N. Brambilla, M. A. Escobedo, J. Ghiglieri, and A. Vairo, Thermal width and gluo-dissociation of quarkonium in pNRQCD, J. High Energy Phys. 12 (2011) 116.

[20] F. Brezinski and G. Wolschin, Gluodissociation and screening of $v$ states in $\mathrm{PbPb}$ collisions at $\sqrt{s_{N N}}=2.76 \mathrm{TeV}$, Phys. Lett. B 707, 534 (2012).
[21] J.-P. Blaizot and E. Iancu, The quark-gluon plasma: Collective dynamics and hard thermal loops, Phys. Rep. 359, 355 (2002).

[22] W. Lucha and F. F. Schoberl, Solving the Schrodinger equation for bound states with MATHEMATICA3.0, Int. J. Mod. Phys. C 10, 607 (1999).

[23] X. Du, R. Rapp, and M. He, Color screening and regeneration of bottomonia in high-energy heavy-ion collisions, Phys. Rev. C 96, 054901 (2017).

[24] M. C. Chu and T. Matsui, Dynamic Debye screening for a heavy anti-quark pair traversing a quark-gluon plasma, Phys. Rev. D 39, 1892 (1989).

[25] M. A. Escobedo, F. Giannuzzi, M. Mannarelli, and J. Soto, Heavy quarkonium moving in a quark-gluon plasma, Phys. Rev. D 87, 114005 (2013).

[26] C. M. Bender and S. A. Orszag, Advanced Mathematical Methods for Scientists and Engineers: I: Asymptotic Methods and Perturbation Theory (Springer, New York, 1999).

[27] S. Kukita, Perturbative dynamics of open quantum systems by renormalization group method, Phys. Rev. E 96, 042113 (2017). 